Homology, Homotopy and Applications, vol.17(2), 2015, pp.161-190

\title{
EQUIVARIANT FIXED-POINT THEORY
}

\author{
KATE PONTO
}

\author{
(communicated by Daniel Dugger)
}

\begin{abstract}
We reexamine equivariant generalizations of the Lefschetz number and Reidemeister trace using categorical traces. This gives simple, conceptual descriptions of the invariants as well as direct comparisons to previously defined generalizations. These comparisons are illuminating applications of the additivity and multiplicativity of the categorical trace.
\end{abstract}

\section{Introduction}

There are two natural ways to approach generalizations of the Lefschetz fixed-point theorem and its converse. One follows the classical description of the Nielsen number $[\mathbf{7}, \mathbf{3 1}, \mathbf{3 2}]$. The alternative approach $[9]$ starts from the more homotopical description of $[6,10,12]$. In this paper we will compare the equivariant invariants arising from these different starting points using formal tools that makes their connections transparent.

The homotopical starting point for equivariant fixed-point theory is a pair of equivariant stable homotopy classes. If $G$ is a finite group, $X$ is a compact $G$-ENR or closed smooth $G$-manifold, and $\{,\}_{G}$ denotes equivariant stable homotopy classes of maps, we associate an equivariant Lefschetz number $L_{G}(f) \in\left\{S^{0}, S^{0}\right\}_{G}$ to every equivariant map $f: X \rightarrow X$. The equivariant Lefschetz number of the identity map is the equivariant Euler characteristic $\chi_{G}(X)$.

If

$$
\Lambda^{f} X:=\left\{\gamma \in X^{I} \mid f(\gamma(0))=\gamma(1)\right\}
$$

is the $f$-twisted loops in $X$, we have an equivariant Reidemeister trace $R_{G}(f) \in$ $\left\{S^{0},\left(\Lambda^{f} X\right)_{+}\right\}_{G}$. For all of these invariants the same notation without a subscript indicates the corresponding nonequivariant object.

Theorem $([\mathbf{6}, \mathbf{9}])$. If $f$ has no fixed points, $L_{G}(f)$ and $R_{G}(f)$ are trivial.

Additionally suppose $X$ is a closed smooth $G$-manifold and for all isotropy subgroups $K \subset H$ of $X \operatorname{dim}\left(X^{H}\right) \geqslant 3$ and $\operatorname{dim}\left(X^{H}\right) \leqslant \operatorname{dim}\left(X^{K}\right)-2$. Then $f$ is equivariantly homotopic to a map with no fixed points if and only if $R_{G}(f)$ is trivial.

The author was partially supported by NSF grants DMS-0703574 and DMS-1207670.

Received August 22, 2014, revised March 18, 2015; published on November 11, 2015.

2010 Mathematics Subject Classification: 18D05, 55M20, 55P25, 55P91.

Key words and phrases: Lefschetz number, Reidemeister trace, fixed point, equivariant homotopy.

Article available at http://dx.doi.org/10.4310/HHA.2015.v17.n2.a9

Copyright (C) 2015, International Press. Permission to copy for private use granted. 
Starting with the Nielsen number, the more natural approach is to look at classical invariants on isotropy subspaces $X^{H}:=\{x \in X \mid x h=x$ for all $h \in H\}$. An equivariant map induces a map $f^{H}: X^{H} \rightarrow X^{H}$ that is equivariant with respect to the action of the Weyl group $W H:=N H / H$. After forgetting the $W H$ action (and ignoring the parts of $X^{H}$ where $W H$ does not act freely), we have a nonequivariant Lefschetz number $L\left(f_{H}\right)$. Alternatively, if the $W H$ action is cellular, we can consider the Hattori-Stallings trace $\operatorname{tr}_{/ W H}$ (Definition 3.9, [24]) of the map induced on the rational cellular chain complex as a map of modules over $W H$. We have analogous options for the Reidemeister trace. In this paper we compare the resulting invariants. In the following two results $G$ is a finite group, $X$ is a closed smooth $G$-manifold, and $f: X \rightarrow X$ is an equivariant endomorphism.

Preliminary Version of Theorem A. If $\operatorname{Conj}(G)$ is a set of representatives for the conjugacy classes of subgroups of $G$,

$$
L_{G}(f)=\sum_{H \in C o n j(G)} \chi_{G}(G / H) \frac{L\left(f_{H}\right)}{\chi(W H)} .
$$

Preliminary Version of Theorem B. There are maps $\mu$ and $\xi$ so that

$$
L_{G}(f)=\sum_{H \in \operatorname{Conj}(G)} \chi_{G}(G / H) \sum_{i}(-1)^{i} \mu\left(\operatorname{tr}_{/ W H}\left(C_{i}\left(f_{H} ; \mathbb{Q}\right)\right)\right)
$$

and $R_{G}(f)$ can be determined from

$$
\left\{\sum_{i}(-1)^{i} \xi\left(\operatorname{tr}_{/\left(\pi_{1}\left(X^{H}\right) \rtimes W H\right)} C_{i}\left(\tilde{f}_{H} ; \mathbb{Q}\right)\right)\right\}_{H \in C o n j}(G)
$$

Later in the paper (at the beginning of Parts 1 and 2) we will be more precise about the maps and give more explicit descriptions of the traces. We will also give more symmetric formulations of the theorems.

Various parts of this theorem can be found in the literature $[\mathbf{7 ,} \mathbf{1 1}, \mathbf{1 3}, \mathbf{2 6}, \mathbf{2 7}$, $\mathbf{2 8}, \mathbf{3 1}, \mathbf{3 2}]$, but we provide a very different proof where much of the hard work is outsourced to formal results for monoidal categories and bicategories $[\mathbf{6 , 1 7 , 1 6 , 2 3 ,}$ 21]. This gives an especially transparent approach that applies to both the Lefschetz number and Reidemeister trace. It is a good example of the advantages of the formal approach.

After briefly recalling the categorical preliminaries, we give proofs of these results in the case that the action in free. Here we make significant use of ideas from the proof of the multiplicativity of traces [23]. In the last three sections we extend to the general case and build on the linearity of traces [21]. The underlying ideas are the same in the second case, but the bookkeeping is more complicated.

Remark 1.1. The different invariants considered here have different natural generalities. Some make sense for compact Lie groups, others infinite discrete groups. In the interest of consistent hypotheses, we will always assume that $G$ is a finite group, but it is useful to remember that this is more restrictive than necessary in some cases. 


\section{Acknowledgments}

I would like to thank Mohammed Abouzaid, Frank Connolly, Peter May, Gun Sunyeekhan, and Bruce Williams for many helpful conversations. I also thank the referee for helpful feedback.

\section{Duality and trace in symmetric monoidal categories}

The trace in symmetric monoidal categories is a generalization of the trace in linear algebra that retains many of the important properties. In particular, it satisfies a generalization of invariance of basis and is functorial. The generalized trace is a trace for endomorphisms of modules over a commutative ring, endomorphisms of chain complexes of modules over a commutative ring, and endomorphisms of closed smooth manifolds or compact ENRs. This section is a summary of $[\mathbf{6}, \mathbf{1 2}, \mathbf{2 0}]$.

Let $\mathscr{V}$ be a symmetric monoidal category with monoidal product $\otimes$, unit $S$, and symmetry isomorphism $\gamma$.

Definition 2.1. An object $A$ in $\mathscr{V}$ is dualizable with dual $B$ if there are morphisms

$$
\eta: S \longrightarrow A \otimes B \quad \epsilon: B \otimes A \longrightarrow S
$$

such that the composites

$$
\begin{aligned}
& A \cong S \otimes A \stackrel{\eta \otimes \mathrm{id}}{\longrightarrow} A \otimes B \otimes A \stackrel{\mathrm{id} \otimes \epsilon}{\longrightarrow} A \otimes S \cong A \\
& B \cong B \otimes S \stackrel{\mathrm{id} \otimes \eta}{\longrightarrow} B \otimes A \otimes B \stackrel{\epsilon \otimes \mathrm{id}}{\longrightarrow} S \otimes B \cong B
\end{aligned}
$$

are identity maps.

We say a space is dualizable if its suspension spectrum is dualizable in the stable homotopy category. A space with a $G$ action is dualizable if its equivariant suspension spectrum is dualizable in the equivariant stable homotopy category.

Proposition 2.2 ([12, III.4.1, III.5.1]). If $X$ is a compact $G$-ENR or closed smooth $G$-manifold, then $X_{+}:=X \amalg *$ is dualizable.

Surprisingly, an explicit description of the dual will not be important in this paper.

Definition 2.3. If $A$ is dualizable with dual $B$ and $f: A \rightarrow A$ is an endomorphism in $\mathscr{V}$, then the trace of $f, \operatorname{tr}(f)$, is the composite

$$
S \stackrel{\eta}{\longrightarrow} A \otimes B \stackrel{f \otimes \text { id }}{\longrightarrow} A \otimes B \stackrel{\gamma}{\longrightarrow} B \otimes A \stackrel{\epsilon}{\longrightarrow} S .
$$

The trace of a chain map is the alternating sum of the levelwise traces. If $f$ is an endomorphism of a topological space and $H_{*}(-: \mathbb{Q})$ is the rational homology functor, the trace of $H_{*}(f: \mathbb{Q})$ is the Lefschetz number of $f$. The trace of an endomorphism of a $G$-space in the equivariant stable homotopy category is the equivariant fixedpoint index $[6]$.

Remark 2.4. In this paper we will generally not distinguish between Lefschetz numbers (computed on the chain complex) and fixed-point indices (computed on the level of spaces) since there are classical identification theorems that show they agree in the cases of interest $[\mathbf{2}, \mathbf{6}]$. These identifications can be made in a way that is compatible with the approach here [17], further reducing the need to make these distinctions. 
In the stable homotopy category and the equivariant stable homotopy category, as well as many other categories, the trace is additive on cofiber sequences.

Theorem $2.5([\mathbf{8}, \mathbf{1 4}])$. In a diagram of cofiber sequences

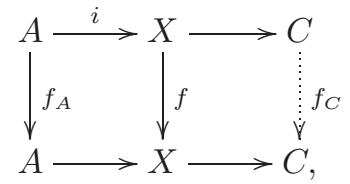

where $A$ and $X$ are dualizable and the left square commutes, $C$ is also dualizable and there is a map $f_{C}$ so that the remaining square commutes and $\operatorname{tr}\left(f_{A}\right)+\operatorname{tr}\left(f_{C}\right)=\operatorname{tr}(f)$.

Spaces with a group action have natural decompositions of this form. If we let $(H)$ denote the conjugacy class of the subgroup $H$ in $G$ and, for a $G$-space $X$ and $x \in X$, $G_{x}:=\{g \in G \mid x g=x\}$, then we use the notation

$$
\begin{gathered}
X_{(H)}:=\left\{x \in X \mid\left(G_{x}\right)=(H)\right\} \quad X^{(H)}:=\left\{x \in X \mid \exists g \in G \text { where } g H g^{-1} \subset G_{x}\right\} \\
X^{>(H)}:=X^{(H)} \backslash X_{(H)} .
\end{gathered}
$$

Each of the inclusion maps $X^{>(H)} \rightarrow X^{(H)}$ is a cofibration [26, II.1.9, II.6.7].

Theorem 2.6 ([26, III.5.4]). If $X$ is a closed smooth $G$-manifold or compact $G$-ENR, then

$$
L_{G}(f)=\sum_{H \in \operatorname{Conj}(G)} L_{G}\left(f_{(H)}\right),
$$

where $f_{(H)}$ is the induced endomorphism of $X^{(H)} / X^{>(H)}$.

Proof. Containment defines a partial order on the set of conjugacy classes of subgroups of $G$. Extend this to a total order

$$
(e)=\left(H_{1}\right)<\left(H_{2}\right)<\left(H_{3}\right)<\cdots<\left(H_{n}\right)=G .
$$

By [26, II.6.7], $X^{\left(H_{i}\right)}$ and $X^{>\left(H_{i}\right)}$ are compact $G$-ENRs and so they are dualizable. Then the map of cofiber sequences

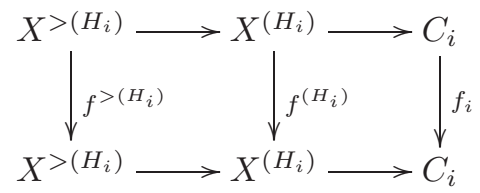

and Theorem 2.5 imply $L_{G}\left(f^{\left(H_{i}\right)}\right)=L_{G}\left(f^{>\left(H_{i}\right)}\right)+L_{G}\left(f_{i}\right)$. In this case we can take $C_{i}=X^{\left(H_{i}\right)} / X_{\left(H_{i}\right)}$ and $f_{i}=f_{\left(H_{i}\right)}$.

Then $L_{G}\left(f^{>\left(H_{k}\right)}\right)$ can be written as a sum of $L_{G}\left(f_{\left(H_{i}\right)}\right)$, where $i \geqslant k$.

In papers such as [30], these types of decompositions play an essential role, but they are expressed in terms of taut maps. A map $f: X \rightarrow Y$ is taut if for all isotropy subgroups $H$ of $X$ there is a neighborhood $V$ of $X^{>H}:=\left\{x \in X \mid H \subsetneq G_{x}\right\}$ in $X^{H}:=$ $\left\{x \in X \mid H=G_{x}\right\}$ and an equivariant retraction $r_{H}: V \rightarrow X^{>H}$ such that $\left.f^{H}\right|_{V}=$ $f^{H} \circ r_{H}$. The assumption that $X$ is a compact $G$-ENR or a closed smooth $G$-manifold 
implies that any equivariant endomorphism of $X$ is equivariantly homotopic to a taut map. Since the invariants here are all defined up to homotopy, taut maps will not play an explicit role in this paper.

\section{Duality and trace in bicategories with shadows}

To define the Reidemeister trace from this perspective and to capture the comparison results in Theorems A and B, we need to extend the trace in a symmetric monoidal category to a bicategory. This section is a brief summary of the relevant parts of $[15,17,19]$.

Definition 3.1. A bicategory $\mathscr{B}$ consists of

- a collection ob: $\mathscr{B}$,

- categories $\mathscr{B}(A, B)$ for each $A, B \in \mathrm{ob} \mathscr{B}$, and

- functors

$$
\begin{gathered}
\odot: \mathscr{B}(A, B) \times \mathscr{B}(B, C) \rightarrow \mathscr{B}(A, C) \\
U_{A}: * \rightarrow \mathscr{B}(A, A)
\end{gathered}
$$

for $A, B$, and $C$ in ob $\mathscr{B}$.

Here $*$ denotes the category with one object and one morphism. The functors $\odot$ are required to satisfy unit and associativity axioms up to natural isomorphisms in $\mathscr{B}(A, B)$.

The elements of ob $\mathscr{B}$ are called $\mathbf{0}$-cells. The objects of $\mathscr{B}(A, B)$ are called $\mathbf{1}$-cells. The morphisms of $\mathscr{B}(A, B)$ are called 2-cells.

Example 3.2. - The 0-cells in the bicategory Mod are rings, and the category $\operatorname{Mod}(R, S)$ for rings $R$ and $S$ is the category of $R$ - $S$-bimodules and their homomorphisms. The composition is given by the tensor product, and a ring regarded as a module over itself is the unit.

- The 0-cells in the bicategory $\mathrm{Ch}$ are rings and the category $\mathrm{Ch}(R, S)$ for rings $R$ and $S$ is the category of chain complexes of $R$-S-bimodules and their chain homotopy classes of maps. The composition is given by the tensor product, and a ring regarded as a module over itself is the unit.

- The 0-cells in the bicategory GpTop are finite groups. A 1-cell $X: G \rightarrow H$ is a based space with an action of $G \times H$ where the actions of $G$ and $H$ are separately free away from the base point. The morphisms from $X: G \rightarrow H$ to $Y: G \rightarrow H$ are stable homotopy classes of equivariant maps from $X$ to $Y$. The bicategorical composition is given by the smash product followed by the quotient by the diagonal action. The unit object associated to a finite group $G$ is $G_{+}$ regarded as a $G-G$ set with a trivial action on the base point.

- The 0-cells in the bicategory Ex of parametrized spectra defined in [15] are topological spaces. The 1-cells are parametrized spectra and the 2-cells are fiberwise stable homotopy classes of maps. The bicategory composition is given by a fiberwise smash product. For this bicategory we will follow the notation and conventions of $[\mathbf{2 3}, \S 3]$. 
There is also a bicategory of parametrized spectra with an action by a finite group $G$.

The first two of these bicategories primarily serve as motivation. Our interest is in bicategories arising in topological settings.

Definition 3.3 ([15, 16.4.1]). A 1-cell $X \in \mathscr{B}(A, B)$ is right dualizable with dual $Y \in \mathscr{B}(B, A)$ if there are 2-cells

$$
\eta: U_{A} \longrightarrow X \odot Y \quad \epsilon: Y \odot X \longrightarrow U_{B}
$$

such that the composites

$$
\begin{aligned}
& Y \cong Y \odot U_{A} \stackrel{\text { id } \odot \eta}{\longrightarrow} Y \odot X \odot Y \stackrel{\epsilon \odot \text { id }}{\longrightarrow} U_{B} \odot Y \cong Y \\
& X \cong U_{A} \odot X \stackrel{\eta \odot \text { id }}{\longrightarrow} X \odot Y \odot X \stackrel{\text { id } \odot \epsilon}{\longrightarrow} X \odot U_{B} \cong X
\end{aligned}
$$

are identity maps.

The map $\eta$ is the coevaluation and $\epsilon$ is the evaluation. We say $(X, Y)$ is a dual pair.

In this paper we will use a range of topological dual pairs. Most are closely related to the classical dual pair in Proposition 2.2.

Theorem 3.4 ([1, 8.6]). If $X$ is a compact ENR or closed smooth manifold with a free right action of a finite group $G$, the space $X_{+}$is dualizable as a* $\times G$ space in the bicategory GpTop.

The evaluation and coevaluation can be interpreted as maps of spaces

$$
S^{n} \rightarrow X_{+} \wedge_{G} D X \text { and } D X \wedge X_{+} \rightarrow G_{+} \wedge S^{n}
$$

for a $G$ space $D X$ and an integer $n[\mathbf{1}]$. The first map is a map of spaces, and the second map is $G$ - $G$-equivariant. To give a better idea of the objects involved, we use the notation $\wedge_{G}$ and $\wedge$ rather than the standard $\odot$ notation. In this case $\wedge_{G}$ is the smash product followed by the quotient by the diagonal $G$ action.

Theorem 3.5 ([17, 3.2.3]). For a closed smooth manifold or compact ENR $X$, the universal cover $\tilde{X}$ is dualizable as a $* \times \pi_{1}(X)$ space in the bicategory GpTop.

We say a parametrized space $E$ over $Y \times X$ is dualizable if the fiberwise suspension spectrum $\Sigma_{Y \times X} E$ is dualizable.

Theorem 3.6 $([\mathbf{1 5}, 18.5 .1,18.6 .1])$. If $X$ is a compact $G$-ENR or closed smooth $G$ manifold, $S_{X}^{0}:=X \amalg X$, regarded as a parametrized space over $* \times X$, is right dualizable.

From a map of topological spaces $f: X \rightarrow Y$, we define spaces $P($ id, $f):=\{(\gamma, x) \in$ $\left.Y^{I} \times X \mid \gamma(0)=f(x)\right\}$ and $P(f$, id $):=\left\{(x, \gamma) \in X \times Y^{I} \mid \gamma(1)=f(x)\right\}$. The first has a map to $Y \times X$ by $(\gamma, x) \mapsto(\gamma(1), x)$, and the second has a similar map to $X \times Y$. These become parametrized spaces with the addition of a disjoint section. We let $Y_{f}:=P(\mathrm{id}, f) \amalg(Y \times X)$ and ${ }_{f} Y:=P(f$, id $) \amalg(X \times Y)$. 
Theorem 3.7 ([15, 17.3.1]). For any map of spaces $f: X \rightarrow Y\left({ }_{f} Y, Y_{f}\right)$ is a dual pair.

Composition of paths and applying the map $f$ to a path define evaluation and coevaluation maps for this dual pair. This type of dual pair will be referred to as a base change dual pair $[\mathbf{1 5}, 17.3]$.

Like the symmetric monoidal trace, the trace of a 2-cell is defined using a composite of the coevaluation and evaluation for a dual pair. Unlike that case, the source of the evaluation and target of the coevaluation are not isomorphic. To accommodate this, we need more structure on a bicategory before we can define the trace.

Definition $3.8([\mathbf{1 7}, 4.4 .1])$. A shadow for a bicategory $\mathscr{B}$ is a functor

$$
\langle-\rangle: \coprod \mathscr{B}(A, A) \rightarrow \mathscr{T}
$$

to a category $\mathscr{T}$ and unital and associative natural isomorphisms $\langle\langle X \odot Y\rangle \cong\langle Y \odot X\rangle$ for every pair of 1-cells $X \in \mathscr{B}(A, B)$ and $Y \in \mathscr{B}(B, A)$.

All of the bicategories in Example 3.2 have shadows [19]. The shadow in GpTop is the quotient by the diagonal action of the group. In the bicategory Ex the shadow is given by pulling back along the diagonal map (up to homotopy) and then quotienting by the resulting section. In particular, for an endomorphism $f: X \rightarrow X$, $\left\langle\left\langle X_{f}\right\rangle \cong\left(\Lambda^{f} X\right)_{+}\right.$.

Definition $3.9([\mathbf{1 7}, 4.5])$. Let $X$ be a dualizable 1 -cell in $\mathscr{B}$ with dual $Y$, and let $f: Q \odot X \rightarrow X \odot P$ be a 2 -cell in $\mathscr{B}$. The trace of $f$ is the composite

$$
\begin{aligned}
\left.\langle Q Q\rangle \cong\left\langle Q \odot U_{A}\right\rangle\right) \stackrel{\text { id } \odot \eta}{\longrightarrow} & \langle Q \odot \odot X \odot Y\rangle \\
& \quad \downarrow \odot \odot \text { id } \\
& \langle X \odot P \odot Y\rangle \stackrel{\sim}{\longrightarrow}\left\langle\langle P \odot Y \odot X\rangle \stackrel{\text { id } \odot \epsilon}{\longrightarrow}\left\langle\left\langle P \odot U_{B}\right\rangle \cong\langle P\rangle\right\rangle .\right.
\end{aligned}
$$

If $M$ is a finitely generated projective right $R$-module, $M$ is right dualizable and the trace of an endomorphism of $M$ is the Hattori-Stallings trace.

If $G$ acts freely on a closed smooth manifold or compact ENR $X$, the trace of an equivariant map $f: X \rightarrow X$ with respect to the dual pair in Theorem 3.4 is a map

$$
\operatorname{tr}_{/ G}(f): S^{n} \rightarrow S^{n} \wedge\left\langle\left\langle G_{+}\right\rangle\right\rangle .
$$

This is another equivariant generalization of the classical fixed-point index. We will see in Theorem B that it is closely related to the equivariant generalization of the index defined in the previous section.

Remark 3.10. If we apply the rational cellular chain complex functor $C_{i}(-; \mathbb{Q})$ to the maps (1), we obtain a dual pair in the bicategory of rings, chain complexes, and homomorphisms. An equivariant map $f: X \rightarrow X$ defines a map of chain complexes, and the trace of this map is

$$
\sum_{i}(-1)^{i} \operatorname{tr}_{/ G}\left(C_{i}(f ; \mathbb{Q})\right),
$$

where $\operatorname{tr}_{/ G}$ is the levelwise Hattori-Stallings trace. This is the universal Lefschetz 
class from [11, 1.7]. By functoriality of the trace [19] this agrees with $\operatorname{tr}_{/ G}(f): S^{n} \rightarrow$ $S^{n} \wedge\left\langle\left\langle G_{+}\right\rangle\right\rangle$under the isomorphism $\pi_{0}^{s}\left(\langle G\rangle_{+}\right) \cong \operatorname{Hom}(\mathbb{Q}, \mathbb{Q}\langle\langle G\rangle)$.

If a space $X$ has a universal cover $\tilde{X}$, an endomorphism $f: X \rightarrow X$ defines an endomorphism $\tilde{f}$ of $\tilde{X}$ that is $\pi_{1}(X)$-equivariant in the sense that for $\alpha \in \pi_{1}(X)$ and $\tilde{x} \in \tilde{X}$

$$
\tilde{f}(x \alpha)=\tilde{f}(x) f_{*}(\alpha)
$$

(with some care with base points). Consistent with notation earlier, we let $\left(\pi_{1} X\right)_{f_{*}+}$ be the set $\left(\pi_{1} X\right)_{+}$with a standard left action of $\pi_{1}(X)$ and a right action of $\pi_{1}(X)$ that is first twisted by $f_{*}$. We can then interpret $f$ as an equivariant map $\tilde{X}_{+} \rightarrow$ $\tilde{X}_{+} \wedge_{\pi_{1}(X)}\left(\pi_{1} X\right)_{f_{*}+}$. The Reidemeister trace of $f, R(f)$, is the bicategorical trace of $\tilde{f}[\mathbf{1 7}]$. It is an element of the zeroth stable homotopy group of the set $\left\langle\pi_{1} X_{f_{*}}\right\rangle:=$ $\pi_{1} X /\left(\gamma f_{*}(\delta) \sim \delta \gamma\right)$.

The map $f: X \rightarrow X$ also defines a fiberwise map

$$
S_{X}^{0} \rightarrow S_{X}^{0} \odot\left(X_{f}\right) .
$$

See [16, 2.3] and [3]. If $X$ is a closed smooth manifold or compact ENR, the trace of this map is an element of the zeroth stable homotopy group of $\left\langle\left\langle X_{f}\right\rangle \cong\left(\Lambda^{f} X\right)_{+}\right.$.

Theorem 3.11 ([16, 4.1]). There is a natural map $\left\langle\left\langle X_{f}\right\rangle \rightarrow\left\langle\left\langle\pi_{1}(X)_{f_{*}}\right\rangle\right.\right.$ and the image of the trace of the fiberwise map $S_{X}^{0} \stackrel{f}{\rightarrow} S_{X}^{0} \odot X_{f}$ under this map is the Reidemeister trace of $f$.

As the natural map $\left\langle\left\langle X_{f}\right\rangle \rightarrow\left\langle\left\langle\pi_{1}(X)_{f_{*}}\right\rangle\right.\right.$ is an isomorphism on components we will follow Remark 2.4 and refer to the trace of $S_{X}^{0} \rightarrow S_{X}^{0} \odot X_{f}$ as the Reidemeister trace of $f$.

If $f: X \rightarrow X$ is an equivariant endomorphism of a closed smooth $G$-manifold or compact $G$-ENR, the equivariant Reidemeister trace of $f$ is defined to be trace of $S_{X}^{0} \rightarrow S_{X}^{0} \odot X_{f}$ in the equivariant parametrized stable homotopy category. This class is denoted $R_{G}(f)$.

Like the symmetric monoidal trace, the bicategorical trace is additive.

Theorem $3.12([\mathbf{2 2}, \mathbf{2 1}])$. Let $A \rightarrow X \rightarrow C$ be a cofiber sequence in the parametrized stable homotopy category or its equivariant generalization, and suppose $A$ and $X$ are dualizable. Then $C$ is also dualizable and, given a commutative diagram

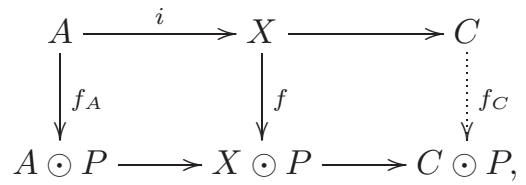

there is a map $f_{C}$ so that the right square commutes and $\operatorname{tr}\left(f_{A}\right)+\operatorname{tr}\left(f_{C}\right)=\operatorname{tr}(f)$.

The approach used in Theorem 2.6 then extends to the Reidemeister trace.

Theorem 3.13 ([21, 6.3]). If $X$ is a closed smooth $G$-manifold or compact $G$-ENR and $f: X \rightarrow X$ is an equivariant endomorphism, then

$$
R_{G}(f)=\sum_{H \in \operatorname{Con} j(G)} i_{(H)} R_{G}\left(f_{(H)}\right) .
$$


Here $R_{G}\left(f_{(H)}\right)$ is the equivariant relative Reidemeister trace of $X^{(H)}$ relative to the subspace $X^{>(H)}$. See [18] and $\S 7$. It is a refinement of the equivariant Reidemeister trace of $X^{(H)} / X^{>(H)}$ and takes values in $\left\{S^{0}, \Lambda^{f^{(H)}} X^{(H)}\right\}_{G}$. The map $i_{(H)}$ is the inclusion $\Lambda^{f^{(H)}} X^{(H)} \rightarrow \Lambda^{f} X$.

We will also use the compatibility of the trace with composites of dual pairs. This was an essential piece of the proofs of multiplicativity [23] and additivity [21].

Theorem $3.14([\mathbf{1 5}, 16.5 .1][\mathbf{2 3}, 5.4])$. If $M \in \mathscr{B}(A, B)$ and $N \in \mathscr{B}(B, C)$ are right dualizable, then so is $M \odot N \in \mathscr{B}(A, C)$.

Let $Q \in \mathscr{B}(A, A), P \in \mathscr{B}(B, B)$, and $R \in \mathscr{B}(C, C)$ be 1-cells, and let $f: Q \odot M \rightarrow$ $M \odot P$ and $g: P \odot N \rightarrow N \odot R$ be 2-cells. Then the following triangle commutes:

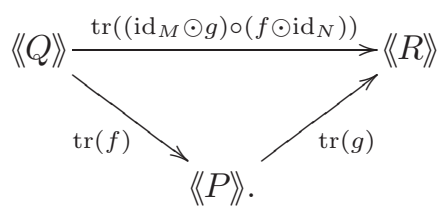

\section{Part 1. Free actions}

An equivariant map $f: X \rightarrow X$ induces a map $\bar{f}: X / G \rightarrow X / G$ so the diagram below commutes:

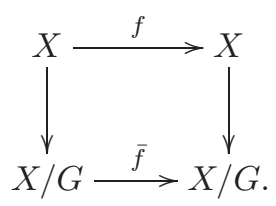

If the action of $G$ on $X$ is free, each $\bar{f}$-twisted loop $\gamma$ in $X / G$ and lift $\tilde{\gamma}$ of $\gamma$ to $X$ define a group element $g \in G$ by $f(\tilde{\gamma}(0))=\tilde{\gamma}(1) g$. Up to conjugacy, this group element depends only on $\gamma$. If $\langle\langle G\rangle$ is the set of conjugacy classes of elements of $G$, we define a map

$$
\Theta: \Lambda^{\bar{f}}(X / G) \rightarrow\langle G\rangle
$$

by $\Theta(\gamma)=g$ if there is a lift $\tilde{\gamma}$ of $\gamma$ such that $f(\tilde{\gamma}(0))=\tilde{\gamma}(1) g$. Let $\operatorname{Fix}(\bar{f})(e):=\Theta^{-1}(e)$. We will also let $\Theta$ denote the corresponding map $\left\langle\left\langle\pi_{1}(X / G)_{\bar{f}_{*}}\right\rangle \rightarrow\langle\langle G\rangle\right.$.

Theorem A (Free case). If action of $G$ on $X$ is free, then $\chi(G) L_{G}(f)=\chi_{G}(G) L(f)$ and there are integers $a_{\gamma}$ so that

$$
R_{G}(f)=\sum_{\gamma \in \operatorname{Fix}(\bar{f})(e)} a_{\gamma}\left(i_{\gamma} \circ \operatorname{tr}_{G}^{\triangle}\left(F_{\gamma}\right)\right) \text { and } R(f)=\sum_{\gamma \in \operatorname{Fix}(\bar{f})(e)} a_{\gamma}\left(i_{\gamma} \circ \operatorname{tr}^{\triangle}\left(F_{\gamma}\right)\right) .
$$

In this statement $\operatorname{tr}^{\triangle}$ is the transfer $[\mathbf{1 2}, 3.7]$. If $X$ is a dualizable object in a symmetric monoidal category, the transfer of $X$ with respect to a map $\triangle: X \rightarrow$ $X \wedge X$ is the composite

$$
S \stackrel{\eta}{\rightarrow} X \wedge D X \stackrel{\triangle \wedge 1}{\longrightarrow} X \wedge X \wedge D X \stackrel{1 \wedge \gamma}{\longrightarrow} X \wedge D X \wedge X \stackrel{1 \wedge \epsilon}{\longrightarrow} X \wedge S \cong X .
$$

For topological spaces with a disjoint base point we will use the map induced by the diagonal. The transfer leads to some unavoidable asymmetry in this statement since 
we cannot multiply by transfers as easily as Euler characteristics. Here $F_{\gamma}$ is the fiber over $\gamma(1)$ and the map $i_{\gamma}: F_{\gamma} \rightarrow \Lambda^{f} X$ is the inclusion of the fiber as constant paths.

For Theorem $\mathrm{B}$ we first need to give a more precise description of the trace we will compare to the Reidemeister trace. Let $\tilde{X}$ be the universal cover of $X$. Then $\tilde{X}$ is a cover of $X / G$ and the action of $\pi_{1}(X / G)$ on $\tilde{X}$ encodes both the $G$ action and the action of $\pi_{1}(X)$. As in the classical case, $\tilde{X}_{+}$is dualizable as a $\pi_{1}(X / G)$ space. An equivariant map $f: X \rightarrow X$ induces a map $\tilde{f}: \tilde{X}_{+} \rightarrow \tilde{X}_{+} \wedge\left(\pi_{1}(X / G)_{\bar{f}_{*}}\right)_{+}$and the trace of $\tilde{f}$ is a map

$$
\operatorname{tr}_{/ \pi_{1}(X / G)}(\tilde{f}): S^{n} \rightarrow S^{n} \wedge\left\langle\left\langle\pi_{1}(X / G)_{\bar{f}_{*}}\right\rangle_{+} .\right.
$$

Both $\operatorname{tr}_{/ G}(f)$ and $\operatorname{tr}_{/ \pi_{1}(X / G)}(\tilde{f})$ are carrying more information than the corresponding classical invariants. To compare invariants we need to be able to separate out this extra information. For each $g \in G$, there is a map $\mu_{g}:\langle G\rangle_{+} \rightarrow S^{0}$ that takes all conjugacy classes of $G$ except the class that contains $g$ to the basepoint. There is also a map $\xi_{g}:\left\langle\pi_{1}(X / G)_{\bar{f}_{*}}\right\rangle_{+} \rightarrow \Theta^{-1}(g)_{+}$that is the identity on $\Theta^{-1}(g)$ and takes all other elements to the basepoint. Let $\zeta_{g}:\left\langle\left\langle\pi_{1}(X)_{f g_{*}}\right\rangle_{+} \rightarrow \Theta^{-1}(g)_{+}\right.$be the composite $\left\langle\left\langle\pi_{1}(X)_{f g_{*}}\right\rangle_{+} \rightarrow\left\langle\left\langle\pi_{1}(X / G)_{\bar{f}_{*}}\right\rangle_{+} \stackrel{\xi_{g}}{\longrightarrow} \Theta^{-1}(g)_{+}\right.\right.$, where the first map is induced by the quotient map $X \rightarrow X / G$.

We abuse notation and let $\xi_{g}$ denote the map $\left(\Lambda^{\bar{f}} X / G\right)_{+} \rightarrow\left\langle\left\langle\pi_{1}(X / G)_{\bar{f}_{*}}\right\rangle_{+} \stackrel{\xi_{g}}{\longrightarrow}\right.$ $\Theta^{-1}(g)_{+}$and let $\zeta_{g}$ denote the map $\left(\Lambda^{f \cdot g} X\right)_{+} \rightarrow\left\langle\left\langle\pi_{1}(X)_{f g_{*}}\right\rangle_{+} \stackrel{\zeta_{g}}{\longrightarrow} \Theta^{-1}(g)_{+}\right.$.

Theorem B (Free case). Let $C_{G}(g)$ be the centralizer of $g$ in $G$. For each $g \in G$

$$
\begin{aligned}
L(f \cdot g) & =\left|C_{G}(g)\right| \mu_{g}\left(\operatorname{tr}_{/ G}(f)\right) \\
\zeta_{g} R(f \cdot g) & =\left|C_{G}(g)\right| \xi_{g}\left(\operatorname{tr}_{/ \pi_{1}(X / G)}(\tilde{f})\right) .
\end{aligned}
$$

Beyond the simplification to the free case, this statement differs from the statement in the introduction in two ways. We can use functoriality of the trace [17] to recover the algebraic descriptions of the traces. (This is the description of the equivariant Reidemeister trace in $[\mathbf{2 7}]$.) We can use Theorem A to recover the original comparison of $\operatorname{tr}_{/ G}$ with $L_{G}$.

The essential underlying observation in this part is that the quotient map $X \rightarrow$ $X / G$ is a covering map. This allows us to use the multiplicativity of the Lefschetz number and Reidemeister trace [23] to express the invariants for $X$ in terms of the invariants for $X / G$ and the fiber. The fibers of $X \rightarrow X / G$ are finite, discrete, and isomorphic to $G$, and the endomorphisms of these fibers induced by an equivariant map of $X$ are determined by their value on a single point. This allows us to easily compute the fiberwise Lefschetz number and fiberwise Reidemeister trace of maps $f: X \rightarrow X$ regarded as maps over $X / G$.

We prove Theorem A in $\S 4$. We prove Theorem B for the Lefschetz number in $\S 5$ and for the Reidemeister trace in $\S 6$. 


\section{Homotopical invariants}

We follow the notation of $[\mathbf{2 3}]$ and use $\left(\widehat{L_{X / G}}\right)(f)$ and $\left(\widehat{R_{X / G}}\right)(f)$ to denote the fiberwise Lefschetz number and Reidemeister trace, respectively. These are stable maps

$$
\left(\widehat{L_{X / G}}\right)(f): \Lambda^{\bar{f}}(X / G) \rightarrow S^{0} \text { and }\left(\widehat{R_{X / G}}\right)(f): \Lambda^{\bar{f}}(X / G) \rightarrow \Lambda^{f}(X) .
$$

Given a path $\gamma$ in $X / G$ from $x$ to $\bar{f}(x)$, we define an endomorphism of the fiber over $\bar{f}(x)$

$$
F_{\bar{f}(x)} \rightarrow F_{x} \stackrel{f}{\rightarrow} F_{\bar{f}(x)},
$$

where the first map is induced by the path $\gamma$. Then $\left(\widehat{L_{X / G}}\right)(f)(\gamma)$ is the Lefschetz number of $(2)$ and $\left(\widehat{R_{X / G}}\right)(f)(\gamma)$ is the Reidemeister trace of $(2)$ composed with the inclusion of the (2) twisted loops in $F_{\bar{f}(x)}$ into the $f$ twisted loops in $X$. The paper [23] considers only the nonequivariant case, but the same approach immediately generalizes to equivariant invariants. We denote these invariants by $\left(\widehat{L_{X / G}}\right)_{G}$ and $\left(\widehat{R_{X / G}}\right)_{G}$.

Proposition 4.1. For an equivariant map $f: X \rightarrow X$ and a $\bar{f}$-twisted loop $\gamma$ in $X / G$,

$$
\begin{gathered}
\left(\widehat{L_{X / G}}\right)(f)(\gamma)= \begin{cases}\chi(G) & \text { if } \Theta(\gamma)=e \\
0 & \text { otherwise }\end{cases} \\
\left(\widehat{L_{X / G}}\right)_{G}(f)(\gamma)= \begin{cases}\chi_{G}(G) & \text { if } \Theta(\gamma)=e \\
0 & \text { otherwise }\end{cases} \\
\left(\widehat{R_{X / G}}\right)(f)(\gamma)= \begin{cases}i_{\gamma} \operatorname{tr}^{\triangle}\left(F_{\gamma}\right) & \text { if } \Theta(\gamma)=e \\
0 & \text { otherwise }\end{cases} \\
\left(\widehat{R_{X / G}}\right)_{G}(f)(\gamma)= \begin{cases}i_{\gamma} \operatorname{tr}_{G}^{\triangle}\left(F_{\gamma}\right) & \text { if } \Theta(\gamma)=e \\
0 & \text { otherwise. }\end{cases}
\end{gathered}
$$

Proof. For a lift $\tilde{\gamma}$ of $\gamma$, the image of $\tilde{\gamma}(1)$ under the first map in (2) is $\tilde{\gamma}(0)$, and so the image under $(2)$ is $f(\tilde{\gamma}(0))=\tilde{\gamma}(1) g$ for some $g \in G$. Since the endomorphism in (2) is equivariant and $F_{\bar{f}(x)}$ is $G$-isomorphic to the $G$-set $G$, the endomorphism is determined by the image of one point and so (2) is multiplication by $g$. In particular, (2) is the identity map if $g$ is the identity element of $G$ and has no fixed points if $g$ is not the identity element of $G$.

The Euler characteristic of $F_{\bar{f}(x)}$ is the same as the Euler characteristic of $G$, so $\left(\widehat{L_{X / G}}\right)(f)(\gamma)=\chi(G)$ and $\left(\widehat{L_{X / G}}\right)_{G}(f)(\gamma)=\chi_{G}(G)$ if $\Theta(\gamma)=e[\mathbf{2 3}, 1.9,6.6]$. Both invariants are zero if $\Theta(\gamma) \neq e$.

The Reidemeister trace of the identity map of a discrete space is the transfer, and the Reidemeister trace of a map with no fixed points is zero. Then $\left(\widehat{R_{X / G}}\right)(f)(\gamma)=$ $i_{\gamma} \operatorname{tr}^{\triangle}\left(F_{\gamma}\right)$ and $\left(\widehat{R_{X / G}}\right)_{G}(f)(\gamma)=i_{\gamma} \operatorname{tr}_{G}^{\Delta}\left(F_{\gamma}\right)$ if $\Theta(\gamma)=e[\mathbf{2 3}, 1.13,7.6]$. Both invariants are zero otherwise. 
Proof of Theorem A (Free case). In [23, 1.17], we showed the triangles
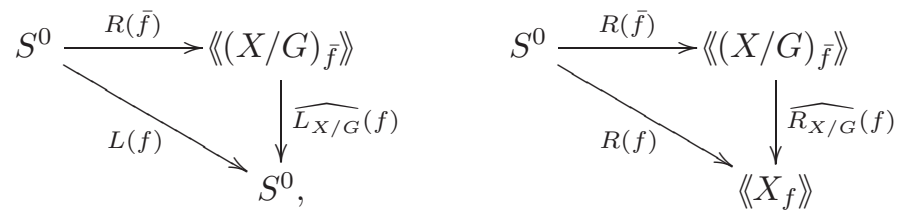

commute. The same approach also shows the corresponding equivariant generalizations commute. (We use the trivial $G$ action on $X / G$, and, if we regard $R(f)$ as an element of $\left\{S^{0}, \Lambda^{\bar{f}} X / G\right\}_{G}$ using the trivial action, we have $\left.R_{G}(\bar{f})=R(\bar{f}).\right)$

By Proposition 4.1 the values of $\chi(G) \cdot\left(\widehat{L_{X / G}}\right)_{G}(f)$ and $\chi_{G}(G) \cdot\left(\widehat{L_{X / G}}\right)(f)$ on any twisted loop in $X / G$ are the same, and so

$$
\begin{aligned}
\chi(G) \cdot L_{G}(f)=\chi(G) \cdot\left(\widehat{L_{X / G}}\right)_{G}(f) \circ R(\bar{f}) & =\chi_{G}(G) \cdot\left(\widehat{L_{X / G}}\right)(f) \circ R(\bar{f}) \\
& =\chi_{G}(G) \cdot L(f) .
\end{aligned}
$$

Using the isomorphism $\left\{S^{0},\left\langle(X / G)_{\bar{f}}\right\rangle_{+}\right\} \cong \mathbb{Z} \pi_{0}\left(\left\langle(X / G)_{\bar{f}}\right\rangle_{+}\right)$, we can write $R(\bar{f})$ as $\sum a_{\gamma} \gamma$, where $a_{\gamma}$ are integers and $\gamma \in \pi_{0}\left(\left\langle(X / G)_{\bar{f}}\right\rangle_{+}\right)$. Then the Reidemeister trace result follows from the second diagram in (3) and Proposition 4.1.

For later results we will need to know a little more about the fiberwise Lefschetz number and Reidemeister trace.

Proposition 4.2. If $\operatorname{Conj}_{G}(h)$ is set of elements of $G$ conjugate to $h$,

$$
\left(\widehat{L_{X / G}}\right)(f \cdot g)(\gamma)= \begin{cases}\left|C_{G}(h)\right| & \text { if } \Theta(\gamma)=h \text { and } g \in \operatorname{Conj}_{G}(h) \\ 0 & \text { otherwise. }\end{cases}
$$

If $x \in X / G$ is a fixed point of $\bar{f}$ and $\tilde{x}$ is a lift of $x$ to $X$ so that $f(\tilde{x})=\tilde{x} h$, then

$$
\left(\widehat{R_{X / G}}\right)(f \cdot g)\left(c_{x}\right)= \begin{cases}\sum_{\{k \in G \mid k=h k g\}} i(\tilde{x} k) & \text { if } g \in \operatorname{Conj}_{G}(h) \\ 0 & \text { otherwise, }\end{cases}
$$

where $i$ is the inclusion of the fixed points of $f \cdot g$ into $\Lambda^{f \cdot g} X$ as constant paths.

Proof. For a $\bar{f}$ twisted loop $\gamma$ in $X / G$ and a lift $\tilde{\gamma}$ of $\gamma$ that satisfies $f(\tilde{\gamma}(0))=\tilde{\gamma}(1) h$, the image of $\tilde{\gamma}(1)$ under the composite

$$
F_{\bar{f}(x)} \rightarrow F_{x} \stackrel{f \cdot g}{\longrightarrow} F_{\bar{f}(x)}
$$

is $\tilde{\gamma}(1) h g$ and the image of $\tilde{\gamma}(1) k$ is $\tilde{\gamma}(1) h k g$.

Since $F_{\bar{f}(x)}$ is discrete, the Lefschetz number is the number of points fixed by the endomorphism. The group action of $G$ on $F_{x}$ is free, so we have a fixed point for each $k \in G$ where $k=h k g$. For any $k \in G$ of this form the map $l \mapsto l k^{-1}$ defines a bijection from the centralizer of $h$ in $G$ to $\{l \in G \mid l=h l g\}$.

The Reidemeister trace is the sum of the constant paths associated to the fixed points. 


\section{Lefschetz numbers for spaces with free actions}

As we see from the previous section, working with parametrized spaces is convenient and powerful. Unfortunately, this approach does not immediately translate to invariants defined using more classical approaches such as $[\mathbf{7}, \mathbf{2 7}, \mathbf{2 8}, \mathbf{3 1}, \mathbf{3 2}]$. To compare the invariants defined here with these alternatives, we will follow the approach in $[\mathbf{1 0}]$ and replace parametrized spaces by spaces with a group action.

To prove Theorem B for the Lefschetz number, we start with a description of $L(f \cdot g)$ in terms of $R(\bar{f})$. Let $\nu_{g}$ be the composite

$$
\Lambda^{\bar{f}}(X / G)_{+} \stackrel{\Theta}{\rightarrow}\left\langle\langle G\rangle_{+} \stackrel{\mu_{g}}{\rightarrow} S^{0} .\right.
$$

Lemma 5.1. For each $g \in G$, the stable map

$$
S^{0} \stackrel{R(\bar{f})}{\longrightarrow}\left\langle(X / G)_{\bar{f}}\right\rangle \cong \Lambda^{\bar{f}}(X / G)_{+} \stackrel{\nu_{g}}{\longrightarrow} S^{0}
$$

is $\frac{1}{\left|C_{G}(g)\right|}(L(f \cdot g))$.

Proof. By Proposition $4.2 \widehat{L_{X / G}}(f \cdot g)$ agrees with the composite

$$
\left\langle(X / G)_{\bar{f}}\right\rangle \stackrel{\nu_{g}}{\longrightarrow} S^{0} \stackrel{\left|C_{G}(g)\right|}{\longrightarrow} S^{0} .
$$

We have a commutative diagram

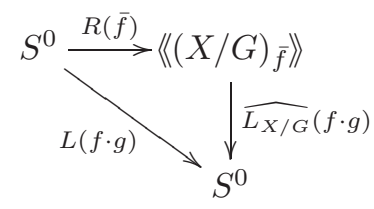

as in (3), and so $L(f \cdot g)=\widehat{L_{X / G}}(f \cdot g) \circ R(\bar{f})=\left|C_{G}(g)\right| \nu_{g} \circ R(\bar{f})$.

To identify $\mu_{g} \circ \operatorname{tr}_{/ G}(f)$ and $\nu_{g} \circ R(\bar{f})$ we first provide another description of the $G$-space $X$. Since $X \rightarrow X / G$ is a covering space, there is a classifying map $\phi: X / G \rightarrow$ $B G$ and a (homotopy) pullback diagram

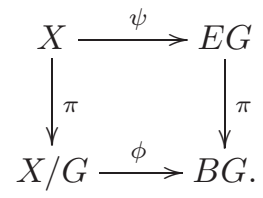

We let $(E G, \pi)$ be $E G \amalg B G$ regarded as a space over $B G \times *$ using $\pi$. In the same way, $(X, \pi)$ is $X \amalg X / G$ regarded as a space over $X / G \times *$. The diagram above defines an equivariant fiberwise equivalence $[\mathbf{2 3}, 3.3]$

$$
(X, \pi) \cong_{\phi}(B G) \odot(E G, \pi)
$$

and $X_{+} \cong S_{X / G}^{0} \odot{ }_{\phi}(B G) \odot(E G, \pi)$. 
Lemma 5.2. There is a fiberwise map $H:(X / G)_{\bar{f}} \bigodot_{\phi}(B G) \rightarrow_{\phi}(B G)$, and the composite

$$
\begin{aligned}
S_{X / G}^{0} \odot{ }_{\phi}(B G) \odot(E G, \pi) & \stackrel{\bar{f} \odot \text { id } \odot \text { id }}{\longrightarrow} S_{X / G}^{0} \odot(X / G)_{\bar{f}} \odot{ }_{\phi}(B G) \odot(E G, \pi) \\
& \stackrel{\text { id } \odot H \odot \text { id }}{\longrightarrow} S_{X / G}^{0} \odot{ }_{\phi}(B G) \odot(E G, \pi) \\
& \stackrel{\text { id } \odot \text { id } \odot(-) g}{\longrightarrow} S_{X / G}^{0} \odot{ }_{\phi}(B G) \odot(E G, \pi)
\end{aligned}
$$

is $f \cdot g: X_{+} \rightarrow X_{+}$.

The fiberwise map $(-) g:(E G, \pi) \rightarrow(E G, \pi)$ is multiplication by $g$.

Proof. If $E G \times E G$ has the diagonal $G$-action, the projection maps $E G \times E G \rightarrow E G$ are $G$-equivariant and so there is a $G$-homotopy $K: E G \times E G \times I \rightarrow E G$ between the projections $[\mathbf{5}, 14.4 .4]$. In the composite

$$
X \times I \stackrel{(f \times \mathrm{id}) \times \mathrm{id}}{\longrightarrow} X \times X \times I \stackrel{\psi \times \psi \times \mathrm{id}}{\longrightarrow} E G \times E G \times I \stackrel{K}{\longrightarrow} E G,
$$

all maps are equivariant, so there is an induced map $H: X / G \times I \rightarrow B G$ that is a homotopy from $\phi \circ \bar{f}$ to $\phi$.

This homotopy defines a map $H: S_{\bar{f}} \odot_{\phi} B G \rightarrow{ }_{\phi} B G$ as in $[\mathbf{2 3}, 3.3]$. The result then follows by explicit computation.

Theorem 3.6 implies $S_{X / G}^{0}$ is right dualizable, and Theorem 3.7 implies ${ }_{\phi} B G$ is right dualizable. To use Lemma 5.2 and Theorem 3.14 to factor the trace we need to describe the dual of $(E G, \pi)$. If $G$ is a finite group, $(E G, \pi)$ is right dualizable [15, 15.1.1], but this is not the dual we need here.

We let $(\overline{E G, \pi})$ denote the fiberwise space $E G \amalg B G$ regarded as a space over $* \times B G$ via $\pi$. Note that $(E G, \pi)$ and $(\overline{E G, \pi})$ both have $G$ actions. We regard $(E G, \pi)$ as a space with a right $G$ action and $(\overline{E G, \pi})$ as a space with a left $G$ action.

Lemma 5.3. There is a $(G \times G)$-equivariant map $(\overline{E G, \pi}) \odot(E G, \pi) \rightarrow G_{+}$and a map $\triangle_{!} S_{B G}^{0}:=\left(B G^{I}, e v_{0} \times e v_{1}\right) \rightarrow(E G, \pi) \wedge_{G}(\overline{E G, \pi})$ over $B G \times B G$ so that the composites as in the definition of a dual pair are homotopic to identity maps through homotopies that respect both the group action and the parameterized structure.

We use $\wedge_{G}$ to indicate quotienting by the diagonal group action after the smash product and $\odot$ to indicate the bicategory composition in Ex.

While these do not define a dual pair in any of the bicategories in Example 3.2, the given structure will allow us to use them in much the same way.

Proof. Define a map $\triangle_{!} S_{B G}^{0} \rightarrow(E G, \pi) \wedge_{G}(\overline{E G, \pi})$ by taking the quotient of the evaluation map $e v_{0} \times e v_{1}: E G^{I} \rightarrow E G \times E G$ by the pointwise action of $G$ on the domain and the diagonal action on the codomain. For each pair $(x, y) \in E G \times_{B G} E G$ there is an element $g$ of $G$ so that $x g=y$. This defines maps $E G \times_{B G} E G \rightarrow G$ and $(\overline{E G, \pi}) \odot(E G, \pi) \rightarrow G_{+}$. 
The image of $(e, \gamma)$ under the composite

$$
(\overline{E G, \pi}) \odot \triangle_{!} S_{B G}^{0} \longrightarrow(\overline{E G, \pi}) \odot(E G, \pi) \odot(\overline{E G, \pi}) \longrightarrow G_{+} \wedge_{G}(\overline{E G, \pi})
$$

is $(g, \tilde{\gamma}(1))$, where $\tilde{\gamma}$ is a lift of $\gamma$ to $E G$ and $g$ is the element of $G$ that takes $e$ to $\tilde{\gamma}(0)$. In particular, if we compose with the isomorphisms $(\overline{E G, \pi}) \cong(\overline{E G, \pi}) \odot \triangle_{!} S_{B G}^{0}$ and $G_{+} \wedge_{G}(\overline{E G, \pi}) \cong(\overline{E G, \pi})$, the map is homotopic to the identity map. The other composite is similar.

Proof of Theorem B (Free Case, Lefschetz Number). As observed above, the maps in Lemma 5.3 are not a coevaluation and evaluation for a dual pair, but we can use Theorem 3.14 and these maps to define a coevaluation and evaluation

$$
S^{n} \rightarrow X_{+} \wedge_{G} D X \quad \text { and } D X \wedge X_{+} \rightarrow G_{+} \wedge S^{n}
$$

as in Theorem 3.4. Since the trace is independent of the choice of dual, we can equally well use this dual to compute $\operatorname{tr}_{/ G}(f)$.

Applying Theorem 3.14 to the decomposition in Lemma 5.2, we see that $\operatorname{tr}_{/ G}(f)$ is the composite

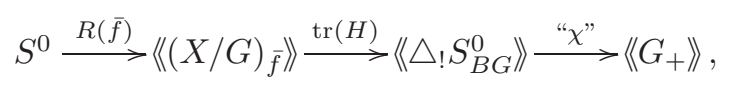

where the rightmost map is defined using the "dual pair" in Lemma 5.3. By $[\mathbf{2 3}, 5.7]$ the image of a twisted loop $\gamma: x \mapsto \bar{f}(x)$ in $X / G$ under $\operatorname{tr}(H)$ is $\phi(\gamma) \cdot \alpha(x)$, where $\alpha$ is the path from $\phi(\bar{f}(x))$ to $\phi(x)$ defined by the homotopy in Lemma 5.2. The map " $\chi$ " lifts this path to $E G$ and assigns the value $g$ if multiplying the initial point of this lift by $g$ is the terminal point of the lift. We obtain the same element by first lifting $\gamma$ to $X$ and a assigning a group element in the same way. Then $\mu_{g} \circ$ " $\chi$ " $\circ \operatorname{tr}(H)=\nu_{g}$ and

$$
\mu_{g} \circ \operatorname{tr}_{/ G}(f)=\mu_{g} \circ " \chi " \circ \operatorname{tr}(H) \circ R(\bar{f})=\nu_{g} \circ R(\bar{f}) \text {. }
$$

\section{Reidemeister traces for spaces with free actions}

We can use very similar ideas to prove the corresponding result for the Reidemeister trace.

Lemma 6.1. For each $g \in G$, the composite

$$
S^{0} \stackrel{R(\bar{f})}{\longrightarrow}\left\langle\left\langle(X / G)_{\bar{f}}\right\rangle \stackrel{\xi_{g}}{\longrightarrow} \Theta^{-1}(g)_{+}\right.
$$

is $\frac{1}{\left|C_{G}(g)\right|} \zeta_{g}(R(f \cdot g))$.

Proof. From Proposition 4.2, if $\tilde{x}$ is a lift of $x$ so that $f(\tilde{x})=\tilde{x} h$,

$$
\left(\widehat{R_{X / G}}\right)(f \cdot g)\left(c_{x}\right)= \begin{cases}\sum_{k \in C_{G}(h)} i_{x}(\tilde{x} k) & \text { if } g \in \operatorname{Conj}_{G}(h) \\ 0 & \text { otherwise. }\end{cases}
$$

When we compose with $\zeta_{h}$, we disregard paths whose value under $\Theta$ is not $h$ and 
we do not distinguish between the paths where $\Theta(\gamma)=h$. In particular,

$$
\zeta_{g} \circ\left(\widehat{R_{X / G}}\right)(f \cdot g)\left(c_{x}\right)= \begin{cases}\left|C_{G}(h)\right| c_{x} & \text { if } g \in \operatorname{Conj}_{G}(h) \\ 0 & \text { otherwise. }\end{cases}
$$

Similarly, $\xi_{h}$ is the identity on paths whose value under $\Theta$ is $h$ and we disregard the other paths. Then $\left|C_{G}(h)\right| \xi_{g}\left(c_{x}\right)=\zeta_{g} \circ\left(\widehat{R_{X / G}}\right)(f \cdot g)\left(c_{x}\right)$ and

$$
\left|C_{G}(h)\right| \xi_{g} \circ R(\bar{f})=\zeta_{g} \circ\left(\widehat{R_{X / G}}\right)(f \cdot g) \circ R(\bar{f})=\zeta_{g} \circ R(f \cdot g) .
$$

To complete the proof of Theorem B it only remains to compare $\xi_{g} \circ \operatorname{tr}_{/ G}(f)$ and $\xi_{g} \circ R(\bar{f})$. This is essentially identical to the proof of the corresponding comparison in the previous section.

Proof of Theorem B (Free Case, Reidemeister Trace). The quotient map $\tilde{X} \rightarrow X \rightarrow$ $X / G$ is classified by a map $\phi: X / G \rightarrow B \pi_{1}(X / G)$, and so we can write $\tilde{X}$ as the composite

$$
S_{X / G}^{0} \odot{ }_{\phi}\left(B \pi_{1}(X / G)\right) \odot\left(E \pi_{1}(X / G), \pi\right)
$$

and $\tilde{f} \cdot g$ as a composite

$$
\begin{aligned}
S_{X / G}^{0} \odot{ }_{\phi}\left(B \pi_{1}(X / G)\right) \odot\left(E \pi_{1}(X / G), \pi\right) \\
\quad \stackrel{\bar{f} \odot \text { id } \odot \text { id }}{\longrightarrow} S_{X / G}^{0} \odot(X / G)_{\bar{f}} \odot{ }_{\phi}\left(B \pi_{1}(X / G)\right) \odot\left(E \pi_{1}(X / G), \pi\right) \\
\quad \stackrel{\text { id } \odot H \odot \text { id }}{\longrightarrow} S_{X / G}^{0} \odot{ }_{\phi}\left(B \pi_{1}(X / G)\right) \odot\left(B \pi_{1}(X / G)\right)_{B(f)} \odot\left(E \pi_{1}(X / G), \pi\right) \\
\quad \stackrel{\text { id } \odot \text { id } \odot(-) g}{\longrightarrow} S_{X / G}^{0} \odot_{\phi}\left(B \pi_{1}(X / G)\right) \odot\left(E \pi_{1}(X / G), \pi\right) \wedge \pi_{1}(X / G)_{\bar{f}_{*}+} \cdot
\end{aligned}
$$

The square

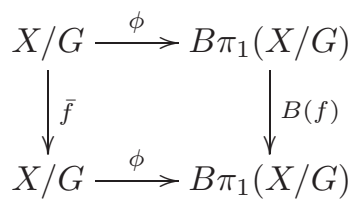

induces a map $H:(X / G)_{\bar{f}} \odot{ }_{\phi}\left(B \pi_{1}(X / G)\right) \rightarrow_{\phi}\left(B \pi_{1}(X / G)\right) \odot\left(B \pi_{1}(X / G)\right)_{B(f)}$ as in $[\mathbf{2 3}, 3.3]$. If $\gamma$ is a path in $B \pi_{1}(X / G)$ so that $\gamma(1)=\pi(e)$, then

$$
(-) g:\left(B \pi_{1}(X / G)\right)_{B(f)} \odot\left(E \pi_{1}(X / G), \pi\right) \rightarrow\left(E \pi_{1}(X / G), \pi\right)
$$

is defined by taking a pair $(\gamma, e)$ to $\gamma_{*}(f(e) g)$, where $\gamma_{*}$ is the map induced on fibers by $\gamma$. This map does not respect the $\pi_{1}(X / G)$ action unless we twist the action on the target by $f$.

The spaces $S_{X / G}^{0}$ and ${ }_{\phi}\left(B \pi_{1}(X / G)\right)$ are right dualizable, and $\left(E \pi_{1}(X / G), \pi\right)$ has a dual as in Lemma 5.3. This gives a factorization of $\operatorname{tr}_{/ \pi_{1}(X / G)}(\tilde{f})$ as the composite

$$
S^{0} \stackrel{R(\bar{f})}{\longrightarrow}\left\langle\left\langle X / G_{\bar{f}}\right\rangle \stackrel{\operatorname{tr}(H)}{\longrightarrow}\left\langle\left(B \pi_{1}(X / G)\right)_{B(f)}\right\rangle\right) \stackrel{\text { " }{ }^{\prime}}{\longrightarrow}\left\langle\left\langle\pi_{1}(X / G)_{f_{*}}\right\rangle_{+},\right.
$$

where the second and third maps take a twisted loop to its homotopy class. Then

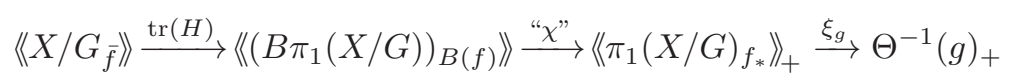


is $\left\langle X X / G_{\bar{f}}\right\rangle \stackrel{\xi_{g}}{\longrightarrow} \Theta^{-1}(g)_{+}$.

\section{Part 2. General actions}

We now generalize to group actions that are not necessarily free. Using Theorems 2.6 and 3.13, to understand $L_{G}(f)$ and $R_{G}(f)$ it is enough to understand the invariants $L_{G}\left(f_{(H)}\right)$ and $R_{G}\left(f_{(H)}\right)$ for each subgroup $H$ of $G$. With this observation, the following statement is the relevant form of Theorem A.

Theorem A (General case). For each subgroup $H$ of $G$,

$$
\chi(W H) L_{G}\left(f_{(H)}\right)=\chi_{G}(G / H) L\left(f_{H}\right)
$$

and there integers $a_{\gamma}$ so that

$$
\begin{gathered}
R_{G}\left(f_{(H)}\right)=\sum a_{\gamma}\left(i_{\gamma} \circ \operatorname{tr}_{G}^{\triangle}\left(F_{\gamma}\right)\right), R\left(f_{(H)}\right)=\sum a_{\gamma}\left(i_{\gamma} \circ \operatorname{tr}^{\triangle}\left(F_{\gamma}\right)\right) \\
R_{W H}\left(f_{H}\right)=\sum a_{\gamma}\left(i_{\gamma}^{\prime} \circ \operatorname{tr}_{W H}^{\triangle}\left(F_{\gamma}^{\prime}\right)\right) \text { and } R\left(f_{H}\right)=\sum a_{\gamma}\left(i_{\gamma}^{\prime} \circ \operatorname{tr}^{\triangle}\left(F_{\gamma}^{\prime}\right)\right),
\end{gathered}
$$

where all sums are taken over $\pi_{0}\left(\Lambda^{f^{H} / W H}\left(X^{H} / W H\right)\right)$.

The equivariant Lefschetz number of the endomorphism of $X^{(H)} / X^{>(H)}$ induced by $f$ is denoted $L_{G}\left(f_{(H)}\right)$, and $L\left(f_{H}\right)$ is the Lefschetz number of the endomorphism of $X^{H} / X^{>H}$ induced by $f$. Here $F_{\gamma}$ is the fiber of $X^{(H)} \rightarrow X^{H} / W H$ over $\gamma(1), i_{\gamma}$ is the inclusion as constant paths, $F_{\gamma}^{\prime}$ is the fiber of $X^{H} \rightarrow X^{H} / W H$ over $\gamma(1)$, and $i_{\gamma}^{\prime}$ is the inclusion of the fiber over $\gamma(1)$ as constant paths.

For Theorem B we need to give a more explicit description of the relevant traces. In Lemma 8.1 we will see that for each subgroup $H$ of $G$ the space $X^{H} \cup C X^{>H}$, the mapping cone of the inclusion $X^{>H} \rightarrow X^{H}$, is dualizable in the bicategory GpTop as a $W H$-space. The trace of the induced map

$$
f^{H}: X^{H} \cup C X^{>H} \rightarrow X^{H} \cup C X^{>H}
$$

is a map $\operatorname{tr}_{/ W H}\left(f^{H}\right): S^{0} \rightarrow\langle W W\rangle_{+}$. As before, this trace is carrying too much information. For each conjugacy class $g$ in $\left\langle\langle W H\rangle_{+}\right.$there is a map $\mu_{W H, g}:\langle W H\rangle_{+} \rightarrow S^{0}$.

In $\S 9$ we will take a very similar trace of an endomorphism of the universal cover of $X^{H}$. The result is a map $\operatorname{tr}_{/ \pi_{1}\left(X^{H}\right) \rtimes W H}\left(f_{H}\right): S^{0} \rightarrow\left\langle\left\langle\left(\pi_{1}\left(X^{H}\right) \rtimes W H\right)_{f^{H}}\right\rangle_{+}\right.$, and there are corresponding quotient maps $\xi_{W H, g}$ and $\zeta_{W H, g}$.

Theorem B (General case). For each $g \in W H$,

$$
\begin{aligned}
L\left(f_{H} \cdot g\right) & =\left|C_{W H}(g)\right| \mu_{W H, g}\left(\operatorname{tr}_{/ W H}\left(f_{H}\right)\right) \\
\zeta_{W H, g} R\left(f_{H} \cdot g\right) & =\left|C_{W H}(g)\right| \xi_{W H, g} \operatorname{tr}_{/ \pi_{1}\left(X^{H}\right) \rtimes W H}\left(\tilde{f}_{H}\right) .
\end{aligned}
$$

One significant difference between the free case and the general case is that from this point on we will restrict to closed smooth manifolds or finite CW complexes. This reflects a single result, Lemma 7.1, which may have a generalization for ENRs. 


\section{Homotopical Invariants}

The proof of Theorem A follows the proof of the corresponding free result but requires a refinement of the relative Reidemeister trace [18]. For a map $Y \rightarrow B$ and $A \subset Y, C_{B}(Y, A)$ is the homotopy pushout of the maps

$$
Y \amalg B \leftarrow A \amalg A \rightarrow A .
$$

This is regarded as a parametrized space over $* \times Y$. The relative Reidemeister trace of a map $f: Y \rightarrow Y$ with $f(A) \subset A$ is the bicategorical trace of the induced map

$$
C_{Y}(Y, A) \rightarrow C_{Y}(Y, A) \odot Y_{f} .
$$

Lemma 7.1. Let $Y$ be a simplicial complex, and let $A \subset Y$ be a subcomplex. There is a neighborhood $U$ of $A$ in $Y$ so that $C_{Y \backslash A}(Y \backslash A, U \backslash A)$ is right dualizable.

It is possible that a similar result would hold for ENRs, but the only proof I know of at this time makes significant use of the simplicial structure.

Proof. Choose a mapping cylinder neighborhood $U$ of $A$ in $Y[4,29]$. There is a retraction of $U \backslash A$ to $\partial(U)$ and a corresponding retraction $Y \backslash A \rightarrow Y^{0}:=Y \backslash \operatorname{Int}(U)$. This gives an equivalence

$$
(Y \backslash A, U \backslash A) \cong\left(Y^{0}, \partial U\right) .
$$

Since $\left(Y^{0}, \partial U\right)$ is a compact $\mathrm{CW}$ pair, it is a compact ENR pair, and [15, 18.5.2] implies that $C_{Y^{0}}\left(Y^{0}, \partial U\right)$ is right dualizable.

If $i: Y^{0} \rightarrow Y \backslash A$ is the inclusion, then $C_{Y^{0}}\left(Y^{0}, \partial U\right) \odot{ }_{i}(Y \backslash A) \simeq C_{Y \backslash A}\left(Y^{0}, \partial U\right)$ [23, p. 1288] and Theorem 3.7 implies that $C_{Y \backslash A}\left(Y^{0}, \partial U\right)$ is right dualizable. By excision $[\mathbf{1 5}, 18.4 .5]$,

$$
C_{Y \backslash A}\left(Y^{0}, \partial U\right) \simeq C_{Y \backslash A}(Y \backslash A, U \backslash A) .
$$

Lemma 7.2. If $A \subset Y$ are closed smooth manifolds and $f: Y \rightarrow Y$ is a continuous map so that $f(A) \subset A$, there is a class in $\pi_{0}^{s}\left(\Lambda^{f}(Y \backslash A)\right)$ so that the image in $\pi_{0}^{s}\left(\Lambda^{f} Y\right)$ is the relative Reidemeister trace of $f$.

Following the notation above, $\Lambda^{f}(Y \backslash A):=\left\{(\gamma, y) \in(Y \backslash A)^{I} \times Y \mid \gamma(1)=f(y)\right\}$. From a classical view of the Reidemeister trace in terms of fixed-point indices and fixed-point classes, this is a very reasonable statement since the constant paths at the fixed points in $Y \backslash A$ are elements of $\pi_{0}\left(\Lambda^{f}(Y \backslash A)\right)$ as well as elements of $\pi_{0}\left(\Lambda^{f} Y\right)$.

Proof. If $i: Y \backslash A \rightarrow Y$ is the inclusion, excision and [15, 18.4.4] imply

$$
C_{Y}(Y, A) \simeq C_{Y \backslash A}(Y \backslash A, U \backslash A) \odot{ }_{i} Y .
$$

The induced map $f: C_{Y}(Y, A) \rightarrow C_{Y}(Y, A) \odot Y_{f}$ defines a map

$$
C_{Y \backslash A}(Y \backslash A, U \backslash A) \odot{ }_{i} Y \longrightarrow C_{Y \backslash A}(Y \backslash A, U \backslash A) \odot{ }_{i} Y \odot Y_{f}
$$

Via adjunction and the dual pair in Theorem 3.7, we have a map

$$
C_{Y \backslash A}(Y \backslash A, U \backslash A) \longrightarrow C_{Y \backslash A}(Y \backslash A, U \backslash A) \odot{ }_{i} Y \odot Y_{f \circ i} .
$$

Theorem 3.14 implies the diagram below where the vertical map is the inclusion of 
paths and $R_{A}(f)$ is the relative Reidemeister trace of $f$ with respect to $A$ commutes:

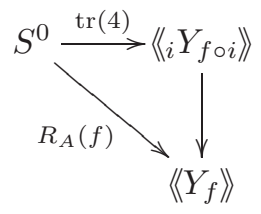

We can lift $R_{A}(f)$ further. Since $A$ is an NDR in $Y$, there is a map $u: Y \rightarrow I$ so that $u^{-1}(0)=A$ and $u^{-1}([0,1))=U$ for some neighborhood $U$ of $A$ in $Y$. Then one choice for (4) is

$$
(y, t) \mapsto\left((f(x), t u(f(y))),\left(f(x), c_{f(x)}, x\right)\right) .
$$

Note that if $y \in Y$ satisfies $f(y) \in A$, then the image of $(y, t)$ is in the section. In fact, the only points whose images are not in the section are those for fixed points in $Y \backslash A$. If $P$ is the subspace $(Y \backslash A) \times_{i}\left(Y^{I}\right) \times_{f}(Y \backslash A)$ consisting of triples where the path lies entirely in $Y \backslash A$ and $P$ is regarded as a space over $(Y \backslash A) \times(Y \backslash A)$, then (4) lifts to define a map

$$
C_{Y \backslash A}(Y \backslash A, U \backslash A) \rightarrow C_{Y \backslash A}(Y \backslash A, U \backslash A) \odot P_{+},
$$

where $P_{+}$is $P$ with a disjoint section added. This allows us to factor the Reidemeister trace of $f$ through the twisted loops in $Y \backslash A$.

Let $i^{H}: X_{H}:=\left\{x \in X \mid G_{x}=H\right\} \rightarrow X^{H}$ denote the inclusion, and let $\pi_{H}: X_{H} \rightarrow$ $X_{H} / W H$ denote the quotient map. If $U^{H}$ is an open neighborhood of $X^{>H}$ in $X^{H}$ that retracts to $X^{>H}$ and

$$
C_{H} X:=C_{X_{H} / W H}\left(X_{H} / W H,\left(U^{H}-X^{>H}\right) / W H\right),
$$

excision implies $[\mathbf{1 5}, 18.4 .5] C_{X^{H}}\left(X^{H}, X^{>H}\right)$ can be written as the composite

$$
C_{H} X \odot\left(X_{H} / W H\right)_{\pi_{H}} \odot{ }_{i}\left(X^{H}\right) .
$$

Lemma 7.3. The map $C_{X^{H}}\left(X^{H}, X^{>H}\right) \rightarrow C_{X^{H}}\left(X^{H}, X^{>H}\right) \odot\left(X^{H}\right)_{f^{H}}$ induced by $f^{H}$ factors as a composite

$$
\begin{aligned}
C_{H} X \odot\left(X_{H} / W H\right)_{\pi_{H}} \odot{ }_{i^{H}}\left(X^{H}\right) \rightarrow C_{H} X \odot P_{+} \odot\left(X_{H} / W H\right)_{\pi_{H}} \odot{ }_{i^{H}}\left(X^{H}\right) \\
\rightarrow C_{H} X \odot\left(X_{H} / W H\right)_{\pi_{H}} \odot i_{i^{H}}\left(X^{H}\right) \odot\left(X^{H}\right)_{f^{H}} \odot\left(X^{H}\right)_{i^{H}} \odot i_{i^{H}}\left(X^{H}\right) \\
\rightarrow C_{H} X \odot\left(X_{H} / W H\right)_{\pi_{H}} \odot{ }_{i^{H}}\left(X^{H}\right) \odot\left(X^{H}\right)_{f^{H}} .
\end{aligned}
$$

Following the notation above, $P:=X_{H} \times_{i^{H}}\left(X_{H}\right)^{I} \times{ }_{f^{H}} X_{H}$.

Proof. The first map is as in the proof of Lemma 7.2, and the last map is the evaluation for the dual pair in Theorem 3.7. 
If $P(f, g)$ is the homotopy pullback of maps $f$ and $g$, we can define a map

$$
\begin{aligned}
P \times_{X_{H} / W H} P\left(\mathrm{id}, \pi_{H}\right) \times_{X_{H}} P\left(i^{H}, \mathrm{id}\right) & \longrightarrow P\left(\mathrm{id}, \pi_{H}\right) \times_{X_{H}} P\left(i^{H}, \mathrm{id}\right) \times_{X^{H}} P\left(\mathrm{id}, f^{H}\right) \\
(\gamma, x),(\beta, y),(y, \alpha) \longmapsto & \longrightarrow(\gamma, f(\tilde{\beta}(0))),(f(\tilde{\beta}(0)), f(\tilde{\beta})),(f(\alpha), \alpha(1)),
\end{aligned}
$$

where $\tilde{\beta}$ is the lift of $\beta$ to a path in $X_{H}$ so that $\tilde{\beta}(1)=y$. This induces a map

$$
P_{+} \odot\left(X_{H} / W H\right)_{\pi_{H}} \odot{ }_{i^{H}}\left(X^{H}\right) \rightarrow\left(X_{H} / W H\right)_{\pi_{H}} \odot{ }_{i}\left(X^{H}\right) \odot\left(X^{H}\right)_{f^{H}} .
$$

Then we have a map

$$
\begin{aligned}
P_{+} \odot\left(X_{H} / W H\right)_{\pi_{H}} & \stackrel{\sim}{\rightarrow} P_{+} \odot\left(X_{H} / W H\right)_{\pi_{H}} \odot \triangle_{!} S_{X_{H}}^{0} / W H \\
& \rightarrow P_{+} \odot\left(X_{H} / W H\right)_{\pi_{H}} \odot i^{H}\left(X^{H}\right) \odot\left(X^{H}\right)_{i^{H}} \\
& \rightarrow\left(X_{H} / W H\right)_{\pi_{H}} \odot i_{i^{H}}\left(X^{H}\right) \odot\left(X^{H}\right)_{f^{H}} \odot\left(X^{H}\right)_{i^{H}},
\end{aligned}
$$

where the first map is an isomorphism, the second is the coevaluation for the dual pair in Theorem 3.7, and the third is defined above.

Note that the same argument applies to the map

$$
C_{X^{(H)}}\left(X^{(H)}, X^{>(H)}\right) \rightarrow C_{X^{(H)}}\left(X^{(H)}, X^{>(H)}\right) \odot\left(X^{(H)}\right)_{f^{(H)}} .
$$

We will also be able to use identical arguments to prove both versions of the Reidemeister trace statements in Theorem A. Because of the remarkable similarity, we only describe the first case.

Proof of Theorem A (General Case). Using Lemma 7.1 we can choose a neighborhood $U^{H}$ of $X^{>H}$ in $X^{H}$ so that $C_{H} X$ is dualizable. Theorem 3.7 implies ${ }_{i(H)} X^{(H)}$ is right dualizable. Since $\pi_{(H)}: X_{(H)} \rightarrow X_{H} / W H$ is a fibration with finite fiber, the space $\left(X_{H} / W H\right)_{\pi_{(H)}}$ is right dualizable by $[\mathbf{2 3}, 4.7]$. Then $C_{X^{(H)}}\left(X^{(H)}, X^{>(H)}\right)$ is right dualizable and Theorem 3.14 and Lemma 7.3 give us a decomposition of the relative Reidemeister trace as the composite

$$
S^{0} \rightarrow\left\langle\langle P\rangle \rightarrow\left\langle_{i(H)} X^{(H)} \odot X_{f^{(H)}}^{(H)} \odot X_{i^{(H)}}^{(H)}\right\rangle \rightarrow\left\langle\left\langle X_{f^{(H)}}^{(H)}\right\rangle\right\rangle,\right.
$$

where the first map is the lift of the relative Reidemeister trace of $\bar{f}^{(H)}$ to the twisted loops in $X_{H} / W H$. The second associates to a twisted loop in $X_{H} / W H$ the Reidemeister trace of the induced endomorphism of the fiber. (There is an induced endomorphism since the path is entirely contained in $X_{H} / W H$ and $X_{(H)} \rightarrow X_{H} / W H$ is a fibration.) The third map is the inclusion of paths.

The computation of the Reidemeister trace of the fiber is as in the proof of Proposition 4.1. Since the factoring applies in both the equivariant and nonequivariant cases, we have the desired decompositions of the Reidemeister trace.

If we choose the integers $a_{x}$ so that $R\left(f_{H} / W H\right)=\sum_{x \in \operatorname{Fix}\left(\bar{f}_{H}\right)} a_{x} c_{x}$ is the relative Reidemeister trace of $f_{H} / W H$ relative to the subspace $X^{>H} / W H$, we have the decomposition in the statement of the theorem. 
If we compose with the collapse maps $\Lambda^{f^{H}} X^{H} \rightarrow *$, identifications of the Reidemeister trace gives corresponding identifications of the Lefschetz number:

$$
\begin{aligned}
L_{G}\left(f_{(H)}\right) & =\sum_{x \in \operatorname{Fix}\left(\overline{f_{H}}\right)(e)} a_{x} \chi_{G}\left(F_{x}\right)=\chi_{G}(G / H) \sum_{x \in \operatorname{Fix}\left(\overline{f_{H}}\right)(e)} a_{x} \\
L\left(f_{(H)}\right) & =\sum_{x \in \operatorname{Fix}\left(\overline{f_{H}}\right)(e)} a_{x} \chi\left(F_{x}\right)=\chi(G / H) \sum_{x \in \operatorname{Fix}\left(\overline{f_{H}}\right)(e)} a_{x}
\end{aligned}
$$

and $\chi(G / H) L_{G}\left(f_{(H)}\right)=\chi_{G}(G / H) L\left(f_{(H)}\right)$.

To replace $L\left(f_{(H)}\right)$ by a multiple of $L\left(f_{H}\right)$, note that

$$
X^{(H)} / X^{>(H)} \cong \bigvee_{K \in C o n j_{G}(H)} X^{K} / X^{>K} \text { and so } L\left(f_{(H)}\right)=\sum_{K \in \operatorname{Conj}_{G}(H)} L\left(f_{K}\right) .
$$

Traces are invariant under cyclic permutation, so if $K$ and $H$ are conjugate, $L\left(f_{K}\right)=$ $L\left(f_{H}\right)$. Then $\chi(W H) L\left(f_{(H)}\right)=\chi(G / H) L\left(f_{H}\right)$, and so

$$
\chi(W H) \chi(G / H) L_{G}\left(f_{(H)}\right)=\chi(W H) \chi_{G}(G / H) L\left(f_{(H)}\right)=\chi(G / H) \chi_{G}(G / H) L\left(f_{H}\right) .
$$

\section{Lefschetz numbers for $G$-spaces}

Much of the work required to prove the general case of Theorem B has been done in the previous section and in the corresponding results in Part 1.

Lemma 8.1. If $X$ is a compact $G$-ENR or closed smooth $G$-manifold, then $X^{H} \cup$ $C X^{>H}$ is dualizable as a WH-space in GpTop.

It is important to note that we cannot expect dualizability for $X^{H}$ in GpTop if the action of $H$ is not free. This is analogous to the algebraic requirement that dualizable modules must be projective.

Proof. If $X$ is a compact $G$-ENR, then $X^{H}$ is a closed $W H$-ENR. As in the proof of Theorem 2.6, $X^{>H}$ is a $W H$-ENR and the inclusion $X^{>H} \rightarrow X^{H}$ is a $W H$-cofibration. Using Theorem 2.5, $X^{H} / X^{>H}$ is $W H$-dualizable and, since the action of $W H$ is free away from the basepoint, $[\mathbf{1}, 8.6]$ implies that $X^{H} / X^{>H}$ is dualizable in GpTop and the dual agrees with the $W H$-equivariant dual.

This statement is essentially the only change we need to make in the proof of the free case of Theorem B for the Lefschetz number.

Proof of Theorem B (General case, Lefschetz number). If $U^{H}$ is a neighborhood of $X^{>H}$ in $X^{H}$ that retracts onto $X^{>H}$ and $U_{H}:=U^{H} \backslash X^{>H}$, then

$$
X^{H} \cup C\left(X^{>H}\right) \simeq X_{H} \cup C\left(U_{H}\right) .
$$

Since the action of $W H$ on $X_{H}$ is free, there is a map $\phi: X_{H} / W H \rightarrow B W H$ that classifies $X_{H} \rightarrow X_{H} / W H$. We can choose $U_{H}$ so that it is $W H$ equivariant and it is classified by the restriction of $\phi$ to $U_{H} / W H$. The result then follows from the proof of the free case if we replace $G$ by $W H$ and $S_{X}^{0}$ by $C_{X_{H} / W H}\left(X_{H} / W H, U_{H} / W H\right)$. 
We can collect these traces $\operatorname{tr}_{/ W H}\left(f_{H}\right)$ into a single trace by generalizing from the category GpTop to the category of profunctors. Associated to a symmetric monoidal category $\mathscr{V}$ with unit $S$ and monoidal product $\otimes$ there is a bicategory $\operatorname{Pro}(\mathscr{V})$ where

- the objects are small categories;

- between two small categories $A$ and $B$ we have the category of functors

$$
A \times B^{\mathrm{op}} \rightarrow \mathscr{V}
$$

and their natural transformations;

- for any small category $A$, there is a functor $U_{A}: A \times A^{\mathrm{op}} \rightarrow \mathscr{V}$ defined by $U_{A}\left(a, a^{\prime}\right)=\amalg_{A\left(a, a^{\prime}\right)} S$;

- for functors $X: A \times B^{\mathrm{op}} \rightarrow \mathscr{V}$ and $Y: B \times C^{\mathrm{op}} \rightarrow \mathscr{V}$, we define $X \odot Y: A \times$ $C^{\text {op }} \rightarrow \mathscr{V}$ by taking $(X \odot Y)(a, c)$ to be the coequalizer of the diagram

$$
\coprod_{b \rightarrow b^{\prime} \in B} X(a, b) \otimes Y\left(b^{\prime}, c\right) \Longrightarrow \coprod_{b \in \mathrm{ob} \mathscr{B}} X(a, b) \otimes Y(b, c),
$$

where the maps are induced by the action of the morphisms of $B$ on $X$ and $Y$; and

- the shadow of $X: A \times A^{\mathrm{op}} \rightarrow \mathscr{V}$ is the coequalizer of the diagram

$$
\coprod_{a \rightarrow a^{\prime} \in \mathrm{ob}(\mathscr{A})} Z\left(a, a^{\prime}\right) \Longrightarrow \coprod_{a \in \operatorname{ob}(A)} Z(a, a) .
$$

The symmetry isomorphism in $\mathscr{V}$ defines the map

$$
\langle X \odot Y\rangle \rightarrow\langle Y \odot \odot X\rangle .
$$

For topological examples we replace coequalizers by homotopy coequalizers using the bar resolution. As in Top, GpTop, and Ex, we say a functor $A \times B^{\mathrm{op}} \rightarrow$ Top is dualizable if the composite with the suspension spectrum functor is dualizable. In some cases this can be described using natural transformations $\eta: S^{n} \wedge A_{+} \rightarrow X \odot Y$ and $\epsilon: Y \odot X \rightarrow S^{n} \wedge B_{+}$in Top so that the usual duality composites are homotopic to the identity after suspension by a sufficiently large sphere.

The generalization of the group $G$ is the component category.

Definition 8.2. The equivariant component category $\Pi_{0}(G, X)$ for a $G$-space $X$ has objects $G$-maps $x(H): G / H \rightarrow X$. The morphisms from $x(H)$ to $y(K)$ are the $G$-maps

$$
\alpha: G / H \rightarrow G / K
$$

such that $y(K) \circ \alpha$ and $x(H)$ are $G$-homotopic.

For any $g \in G$ so that $g^{-1} H g \subset K$, there is a $G$-map $R_{g}: G / H \rightarrow G / K$ defined by $R_{g}(l H)=l g K$. All $G$-maps $G / H \rightarrow G / K$ are of this form, and two such maps $R_{g}$ and $R_{h}$ are the same only if $g h^{-1} \in K$.

If $x(H): G / H \rightarrow X$ is a $G$-map and $C_{x}$ is the component of $X^{H}$ that contains $x(e H)$, let $X^{H}(x)$ be the pullback of the quotient map $X^{H} \rightarrow X^{H} / W H$ and the 
inclusion $\left(W H C_{x}\right) / W H \rightarrow X^{H} / W H$. If $X^{>H}(x):=\left\{y \in X^{H}(x) \mid H \subsetneq G_{y}\right\}$, define a functor

$$
\overline{G \mid X}: \Pi_{0}(G, X)^{\mathrm{op}} \rightarrow \text { Top }
$$

by $\overline{G \mid X}(x(H))=X^{H}(x) / X^{>H}(x)$. On morphisms we use the induced group action.

Proposition 8.3. If $X$ is a compact $G$-ENR or closed smooth $G$-manifold, then $\overline{G \mid X}$ is dualizable.

Proof. Using $[\mathbf{1 8}, 3.7]$, it is enough to show that $\overline{G \mid X}(x(H))$ is dualizable for each object $x(H)$ relative to the action by $\Pi_{0}(G, X)(x(H), x(H))$. This is Lemma 8.1.

Given an equivariant map $f: X \rightarrow X$, let $\Pi_{0}^{f}(G, X)$ be the functor $\Pi_{0}(G, X) \times$ $\Pi_{0}(G, X)^{\mathrm{op}} \rightarrow$ Top defined by

$$
\Pi_{0}^{f}(G, X)(x(H), y(K)):=\Pi_{0}(G, X)(f(y(K)), x(H)) .
$$

Then an endomorphism $f: X \rightarrow X$ induces a natural transformation

$$
\bar{f}: \overline{G \mid X} \rightarrow \overline{G \mid X} \odot \Pi_{0}^{f}(G, X) .
$$

The trace of $\bar{f}$ is a map $S^{0} \rightarrow\left\langle\left\langle\Pi_{0}^{f}(G, X)\right\rangle\right.$.

Proposition 8.4. If $W H_{x, f}:=\left\{g \in W H \mid[f(x) g]=[x] \in \pi_{0}\left(X^{H}\right)\right\}$ and $B(X)$ is the isomorphism classes of objects of $\Pi_{0}(G, X)$, there is an isomorphism

$$
\delta:\left\langle\Pi_{0}^{f}(G, X)\right\rangle_{+} \rightarrow \coprod_{x(H) \in B(X)}\left\langle\left\langle W H_{x, f}\right\rangle_{+} .\right.
$$

If $\delta_{x(H), f}:\left\langle\Pi_{0}^{f}(G, X)\right\rangle_{+} \rightarrow\left\langle W W H_{x, f}\right\rangle_{+}$is one of the projections, the composite

$$
\left.S^{0} \stackrel{\operatorname{tr}(\bar{f})}{\longrightarrow}\left\langle\left\langle\Pi_{0}^{f}(G, X)\right\rangle\right) \stackrel{\delta_{x(H), f}}{\longrightarrow}\left\langle W H_{x, f}\right\rangle\right\rangle
$$

is $\operatorname{tr} / W H(f)$.

Proof. Using the identifications

$$
\begin{aligned}
\Pi_{0}(G, X)(f(x(H)), x(H)) & =\left\{R_{g}: G / H \rightarrow G / H \mid f(x(H)) \circ R_{g} \sim_{G} x(H)\right\} \\
& =\left\{g \in W H \mid[f(x(H)) g]=[x(H)] \in \pi_{0}\left(X^{H}\right)\right\},
\end{aligned}
$$

we have the isomorphism $\delta$ above. Then $[\mathbf{1 8}, 3.6,3.7]$ completes the proof.

We can also compose with the cellular chain complex functor to define a functor

$$
C_{*}(\overline{G \mid X}): \Pi_{0}(G, X)^{\mathrm{op}} \rightarrow \mathrm{Ch}_{\mathbb{Q}} .
$$

If $X$ is a compact $G$-ENR or closed smooth $G$-manifold, this functor is dualizable by functoriality. The natural transformation $\bar{f}: \overline{G \mid X} \rightarrow \overline{G \mid X} \odot \Pi_{0}^{f}(G, X)$ induces a natural transformation

$$
\bar{f}_{*}: C_{*}(\overline{G \mid X}) \rightarrow C_{*}(\overline{G \mid X}) \odot \mathbb{Q} \Pi_{0}^{f}(G, X),
$$

where $\mathbb{Q} \Pi_{0}^{f}(G, X)$ is is the functor $\Pi_{0}(G, X) \times \Pi_{0}(G, X) \rightarrow \mathrm{Ch}_{\mathbb{Q}}$ defined by

$$
\mathbb{Q} \Pi_{0}^{f}(G, X)(x(H), y(K)):=\mathbb{Q} \Pi_{0}(G, X)(f(y(K)), x(H)) .
$$

Functoriality of the trace implies the trace of (6) agrees with the trace of (5). The following result is an immediate consequence. 
Theorem 8.5. If $f: X \rightarrow X$ is an equivariant map and the set

$\{x \in X \mid$ there is $g \in G$ such that $f(x)=x g\}$

is empty, then the trace of (6) is trivial.

\section{Reidemeister traces for $G$-spaces}

We can now combine $\S 6$ and $\S 8$ and give another description of the Reidemeister trace for spaces with a group action that is not necessarily free. We start with the common generalization of the fundamental group and the component category.

Definition 9.1. The objects of the equivariant fundamental category $\Pi(G, X)$ of a $G$-space $X$ are the $G$-maps $x(H): G / H \rightarrow X$. A morphism from $x(H)$ to $y(K)$ is a $G$-map

$$
R_{g}: G / H \rightarrow G / K
$$

and a homotopy class of $G$-maps

$$
w(H): G / H \times I \rightarrow X
$$

relative to $G / H \times \partial I$ such that $w(H)(-, 0)=x(H)$ and $w(H)(-, 1)=y(K) \circ R_{g}$.

The composite of $\left(R_{g}, w(H)\right)$ and $\left(R_{h}, v(K)\right)$ is $\left(R_{h} \circ R_{g},\left(v(K) \circ R_{g}\right) w(H)\right)$.

Let $\widetilde{X^{H}}(x)$ be the universal cover of $X^{H}(x)$. The usual action of paths on the cover defines an action of an endomorphism $\left(R_{g}, w(H)\right)$ of $x(H)$ in $\Pi(G, X)$ on a point $\tilde{x}$ in $\widetilde{X^{H}}(x)$ by $\tilde{x} \mapsto(\tilde{x} g) \cdot w(H)(e)$. Let $\overline{X^{>H}}(x)$ be the pullback of $\widetilde{X^{H}}(x)$ along the inclusion $X^{>H} \rightarrow X^{H}$, and let $\overline{X_{H}}(x)$ be the pullback of $\widetilde{X^{H}}(x)$ along the inclusion $X_{H} \rightarrow X^{H}$. The group action of $\Pi(G, X)(x(H), x(H))$ defines a group action on $\overline{X_{H}}(x)$. Define a functor

$$
\hat{X}: \Pi(G, X) \rightarrow \text { Top }
$$

by $\hat{X}(x(H)):=\widetilde{X^{H}}(x) \cup C\left(\overline{X^{>H}}(x)\right)$.

Lemma 9.2. If $X$ is a compact $G$-ENR or a closed smooth $G$-manifold, then $\hat{X}(x(H))$ is dualizable in GpTop as a $\Pi(G, X)(x(H), x(H))$-space.

Proof. There is a diagram

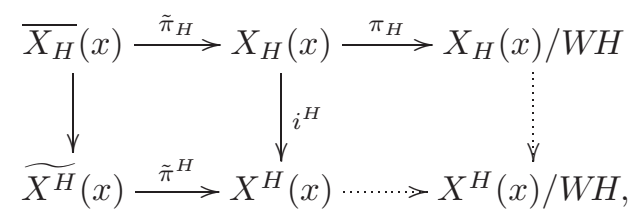

where the vertical maps are inclusions and the horizontal maps are quotients. The top corner is a pullback square and hence a homotopy pullback since $\widehat{X^{H}} \rightarrow X^{H}$ is a 
covering map. If $\pi: X_{H}(x) \rightarrow *$, then

$$
\begin{aligned}
\left(X_{H}(x) / W H\right)_{\pi_{H}} \odot_{i^{H}} X^{H}(x) \odot\left(X^{H}(x)\right)_{\tilde{\pi}^{H}} \odot \pi^{*} & \left.\simeq\left(X_{H}(x) / W H\right)_{\pi_{H} \tilde{\pi}_{H}}\right) \odot_{\pi^{*}} \\
& \simeq\left(\overline{X_{H}}\left(\overline{x), \pi_{H}} \tilde{\pi}_{H}\right)_{+} .\right.
\end{aligned}
$$

There is an isomorphism

$$
\begin{gathered}
\Pi(G, X)(x(H), x(H)) \longrightarrow \pi_{1}\left(X^{H}\right) \rtimes W H \\
\left(R_{g}, w(H)\right) \longrightarrow(w(e H), g),
\end{gathered}
$$

and this is compatible with the actions of each group on $\overline{X_{H}}(x)$. The composite of quotient maps $\overline{X_{H}}(x) \rightarrow X_{H}(x) \rightarrow X_{H}(x) / W H$ is the quotient by the action of $\pi_{1}\left(X^{H}\right) \rtimes W H$. Then we can use the approach of Lemma 5.3 to define maps

$$
\begin{gathered}
\triangle_{!} S_{X_{H} / W H}^{0} \rightarrow\left(\overline{X_{H}}\left(\overline{x), \pi_{H}} \tilde{\pi}_{H}\right) \wedge_{\pi_{1}\left(X^{H}\right) \rtimes W H}\left(\overline{X_{H}}(x), \pi_{H} \tilde{\pi}_{H}\right)\right. \\
\left(\overline{X_{H}}(x), \pi_{H} \tilde{\pi}_{H}\right) \odot\left(\overline{X_{H}}\left(\overline{x), \pi_{H}} \tilde{\pi}_{H}\right) \rightarrow\left(\pi_{1}\left(X^{H}\right) \rtimes W H\right)_{+}\right.
\end{gathered}
$$

so that the required triangle diagrams commute.

Using the decomposition before Lemma 7.3 and [15, 18.4.4],

$$
\begin{aligned}
\widetilde{X^{H}}(x) \cup & C\left(\overline{X^{>H}}(x)\right) \simeq C_{\widetilde{X^{H}}(x)}\left(\widetilde{X^{H}}(x), \overline{X^{>H}}(x)\right) \odot \pi^{*} \\
& \simeq C_{X^{H}(x)}\left(X^{H}(x), X^{>H}(x)\right) \odot\left(X^{H}(x)\right)_{\tilde{\pi}^{H}} \odot \pi^{*} \\
& \simeq C_{H} X(x) \odot\left(X_{H}(x) / W H\right)_{\pi_{H}} \odot_{i^{H}} X^{H}(x) \odot\left(X^{H}(x)\right)_{\tilde{\pi}^{H}} \odot{ }_{\pi} * .
\end{aligned}
$$

From Lemma $7.1 C_{H} X(x):=C_{X_{H}(x) / W H}\left(X_{H}(x) / W H,\left(U^{H}-X^{>H}\right) / W H\right)$ is right dualizable. The remaining parts of the decomposition are dualizable by the discussion above.

Lemma 9.2 extends in the same way that Proposition 8.3 follows from Lemma 8.1.

Proposition 9.3. If $X$ is a compact $G$-ENR, then $\hat{X}$ is dualizable as a right $\Pi(G, X)$ space.

Let $\Pi^{f}(G, X)$ be the functor $\Pi(G, X)-\Pi(G, X)^{\text {op }} \rightarrow$ Top defined by

$$
\Pi^{f}(G, X)(x(H), y(K)):=\Pi(G, X)(f(y(K)), x(H)) .
$$

Then an equivariant map $f: X \rightarrow X$ defines a natural transformation $\tilde{f}: \hat{X} \rightarrow \hat{X} \odot$ $\Pi^{f}(G, X)$. The trace of $\tilde{f}$ is a map $\left\langle\left\langle S^{0}\right\rangle\right\rangle \rightarrow\left\langle\Pi^{f}(G, X)\right\rangle$.

The following result is a consequence of $[\mathbf{1 8}, 3.6,3.7]$.

Proposition 9.4. There is an isomorphism

$$
\left\langle\left\langle\Pi^{f}(G, X)\right\rangle \cong \coprod\left\langle\Pi^{f}(G, X)\right\rangle(x(H), x(H)),\right.
$$

where the coproduct is taken over a choice of representatives of the isomorphism classes of objects of $\Pi(G, X)$. The image of $\operatorname{tr}(\tilde{f})$ under the projection

$$
\coprod\left\langle\langle \Pi ^ { f } ( G , X ) \rangle ( x ( H ) , x ( H ) ) \rightarrow \left\langle\left\langle\Pi^{f}(G, X)\right\rangle(x(H), x(H))\right.\right.
$$

is the trace of the induced map $\hat{X}(x(H)) \rightarrow \hat{X}(x(H)) \odot \Pi^{f}(G, X)(x(H), x(H))$ in GpTop with respect to the group action by $\Pi(G, X)(x(H), x(H))$. 
Exactly as before, we can compose the dual pair for $\hat{X}$ with the cellular chain complex functor and define algebraic invariants. This is the refined equivariant Lefschetz number from $[\mathbf{2 7}, 5.7]$. Using functoriality of the trace, this agrees with the topologically defined trace of $\tilde{f}: \hat{X} \rightarrow \hat{X} \odot \Pi^{f}(G, X)$.

We can now finish the proof of Theorem B. The map $\Theta$ defined in the Part 1 extends to a map

$$
\Theta_{W H}:\left\langle\left\langle\Pi^{f}(G, X)(x(H), x(H))\right\rangle \rightarrow\langle\langle W H\rangle\rangle\right.
$$

by $\Theta_{W H}\left(R_{g}, w\right)=g$. Let

$$
\left\langle\left\langle\Pi^{f}(G, X)(x(H), x(H))\right\rangle_{+} \stackrel{\xi_{W H, g}}{\longrightarrow} \Theta_{W H}^{-1}(g)_{+} \text {and }\left\langle\pi_{1}\left(X^{H}\right)_{g f_{*}^{H}}\right\rangle_{+} \stackrel{\zeta_{W H, g}}{\longrightarrow} \Theta_{W H}^{-1}(g)_{+}\right.
$$

be collapse maps generalizing the maps $\xi_{g}$ and $\zeta_{g}$.

Proof of Theorem B (General case, Reidemeister trace). We can use essentially the same proof as in the case of a free action.

The trace $\operatorname{tr}_{/ \pi_{1}\left(X^{H}\right) \rtimes W H}\left(\tilde{f}_{(H)}\right)$ is the trace of the map

$$
\widetilde{X^{H}}(x) \cup C\left(\overline{X^{>H}}(x)\right) \rightarrow\left(\widetilde{X^{H}}(x) \cup C\left(\overline{X^{>H}}(x)\right)\right) \wedge_{\pi_{1}\left(X^{H}\right) \rtimes W H}\left(\pi_{1}\left(X^{H}\right) \rtimes W H\right)_{f_{*}^{H}}
$$

induced by $f^{H}$. Using the discussion above, $\widetilde{X^{H}}(x) \cup C\left(\overline{X^{>H}}(x)\right)$ is the composite $C_{H} X(x) \odot\left(X_{H}(x) / W H\right)_{\pi_{H}} \odot{ }_{i^{H}} X^{H}(x) \odot\left(X^{H}(x)\right)_{\tilde{\pi}^{H}} \odot \pi^{*}$. We can replace this by $C_{H} X(x) \odot\left(X_{H}(x) / W H\right)_{\pi_{H}} \odot\left(X_{H}(x)\right)_{\tilde{\pi}_{H}} \odot \tilde{i}^{H} \overline{X^{H}}(x) \odot \pi^{*}$, where $\tilde{i}^{H}$ is the induced map $\overline{X^{H}}(x) \rightarrow \widehat{X^{H}}(x)$. We can further simplify to $C_{H} X(x) \odot\left(X_{H}(x) / W H\right)_{\pi_{H} \circ \tilde{\pi}_{H}} \odot$ $\pi \circ \tilde{i}^{H} *$.

There is a map $\phi: X_{H}(x) / W H \rightarrow B\left(\pi_{1}\left(X^{H}\right) \rtimes W H\right)$ and a pullback diagram

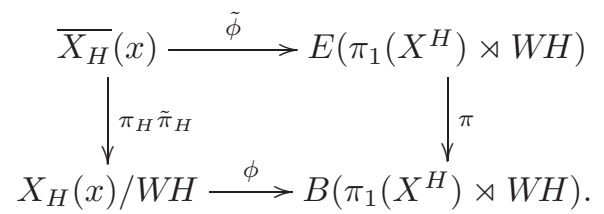

Then

$$
\begin{aligned}
{ }_{\phi} B\left(\pi_{1}\left(X^{H}\right) \rtimes W H\right) \odot B\left(\pi_{1}\left(X^{H}\right) \rtimes W H\right)_{\pi} \\
\cong\left(X_{H}(x) / W H\right)_{\pi_{H} \tilde{\pi}_{H}} \odot_{\tilde{\phi}} E\left(\pi_{1}\left(X^{H}\right) \rtimes W H\right) .
\end{aligned}
$$

Using the identifications $B\left(\pi_{1}\left(X^{H}\right) \rtimes W H\right)_{\pi} \odot{\tilde{\phi} \circ \pi \circ \tilde{i}^{H}}^{*} \cong\left(E\left(\pi_{1}\left(X^{H}\right) \rtimes W H\right), \pi\right)$ and $\tilde{\phi} E\left(\pi_{1}\left(X^{H}\right) \rtimes W H\right) \odot_{\pi \circ \tilde{i}^{H}} * \cong \tilde{\phi}_{\tilde{\phi} \pi \pi^{\circ} \tilde{i}^{H}} *$, we can write $\widetilde{X^{H}}(x) \cup C\left(\overline{X^{>H}}(x)\right)$ as

$$
C_{H} X(x) \odot{ }_{\phi} B\left(\pi_{1}\left(X^{H}\right) \rtimes W H\right) \odot\left(E\left(\pi_{1}\left(X^{H}\right) \rtimes W H\right), \pi\right) .
$$

As in the free case, there is a corresponding decomposition of the map induced by $f$ and we have the factoring of the trace.

\section{Equivariant Nielsen numbers}

Fadell and Wong [7] and Wilczyński [30] have given very different proofs of the converse to the equivariant Lefschetz fixed-point theorem. They used generalizations 
of the Nielsen number. We can compare their invariant with the equivariant Reidemeister trace using the results above. We start with a consequence of Theorem A.

Proposition 10.1. $R_{G}(f)$ is zero if and only if $R\left(f^{H}\right)$ is zero for all subgroups $H$ of $G$.

Let Iso $(X)$ be a choice of representatives for the isomorphism classes of objects in $\Pi_{0}(G, X)$. Without loss of generality we may assume that we have first chosen representatives for each conjugacy class of subgroups of $G$ and that only these representatives appear among the objects of Iso $(X)$. Let Iso $(X)(H)$ be the isomorphism classes of objects associated to maps $G / H \rightarrow X$.

Lemma 10.2. For each subgroup $H$ of $G$ the forgetful map $\mathbb{Z I s o}(X)(H) \rightarrow \mathbb{Z} \pi_{0}\left(X^{H}\right)$ defined by $x(H) \mapsto \sum_{g H \in W H / H} x(g H)$ is injective.

Proof. The image of each $x(H)$ is nontrivial since all terms appear with coefficient 1 .

Suppose that $x(g H)$ and $y\left(g^{\prime} H\right)$ are in the same component of $X^{H}$. Then $x(H)$ and $g^{-1} g^{\prime} y(H)$ are in the same component of $X^{H}$, and, in particular, $x(H)$ and $y(H)$ represent isomorphic objects in Iso $(X)(H)$.

Proof of Proposition 10.1. The zeroth equivariant stable homotopy group of a $G$ space $X_{+}$is the free abelian group generated by $x(H) \circ \operatorname{tr}_{G}^{\triangle}(G / H)$ for $x(H) \in \operatorname{Iso}(X)$ $[\mathbf{5}, 2.8 .13 .7]$. Combining coefficients if necessary, we can use Theorem A to express $R_{G}(f)$ as a sum

$$
\sum_{\gamma \in \mathbf{I s o}\left(\Lambda^{f} X\right)} a_{\gamma}\left(i_{\gamma} \circ \operatorname{tr}_{G}^{\triangle}\left(F_{\gamma}\right)\right)
$$

where each element in Iso $\left(\Lambda^{f} X\right)$ is associated to exactly one coefficient. Then $R_{G}(f)$ is zero if and only if each of the $a_{\gamma}$ are zero.

Additivity of the Reidemeister trace implies that $R\left(f^{H}\right)$ is zero for all subgroups $H$ of $G$ if and only if $R\left(f_{H}\right)$ is zero for all subgroups $H$ of $G$. Then Theorem A allows us to conclude that

$$
R\left(f_{H}\right)=\sum a_{\gamma}\left(i_{\gamma}^{\prime} \circ \operatorname{tr}^{\triangle}\left(F_{\gamma}^{\prime}\right)\right)
$$

where we have exactly the same coefficients $a_{\gamma}$ as above and we sum over the elements of Iso $\left(\Lambda^{f} X\right)(H)$.

The invariant $R\left(f_{H}\right)$ is the image of $R_{W H}\left(f_{H}\right)$ under the forgetful map, and so Lemma 10.2 implies $R\left(f_{H}\right)$ is zero if and only if each of the $a_{\gamma}$ are zero.

Recall that two fixed points $x$ and $y$ in $X^{H}$ are in the same fixed-point class if the images of the constant paths at $x$ and $y$ are in the same component of $\Lambda^{f^{H}} X^{H}$. We say $x$ and $y$ are in the same $W H$-fixed-point class if there is a $g \in W H$ so that the constant paths at $x$ and $y g$ are in the same component of $\Lambda^{f^{H}} X^{H}$. Note that there is a map from the fixed-point classes to the $W H$-fixed-point classes.

If $H$ is subconjugate to $K$, the inclusion induces a map $\tau_{H \leqslant K}$ from the $W K$-fixedpoint classes to the $W H$-fixed-point classes. We let $W H$-fpc denote the set of $W H$ fixed-point classes, and for $\alpha \in W H$-fpc we let $i\left(f^{H}, \alpha\right)$ denote the nonequivariant fixed-point index of the fixed points in the class $\alpha$ with respect to the map $f^{H}: X^{H} \rightarrow$ $X^{H}$. (At this point it is convenient to replace $f$ by a homotopic map that it taut and has isolated fixed points. See $\S 2$.) 
Definition 10.3 ([31]). The equivariant Nielsen number of $f, N_{G}(f)$, is the function from the conjugacy classes of subgroups of $G$ to the integers defined by

$$
N_{G}(f)(H)=\sharp\left\{\begin{array}{l|l}
\alpha \in W H \text {-fpc } & \begin{array}{c}
i\left(f^{H}, \alpha\right) \neq 0 \text { and } i\left(f^{K}, \delta\right)=0 \text { for all } \\
\delta \in W K \text {-fpc so that } \tau_{H \leqslant K}(\delta)=\alpha
\end{array}
\end{array}\right\} .
$$

Note that the equivariant Nielsen number is not the number of generators in the equivariant Reidemeister trace with nonzero coefficient. The equivariant Nielsen number is a nonredundant count of the number of nonzero coefficients. In particular, the coefficients of equivariant Reidemeister trace do not give a lower bound for the number of fixed points.

Theorem 10.4. The equivariant Nielsen number of a map is zero if and only if the equivariant Reidemeister trace is zero.

Proof. For each $g \in W H$ the diagram

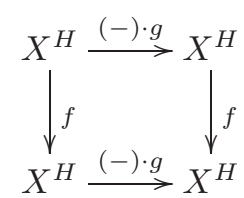

commutes, and so for each fixed point $x$ of $f^{H}$, the indices of $x$ and $x g$ are the same [2, IV.B.7]. This implies that a $W H$-fixed-point class can only have index zero if all the associated (classical) fixed-point classes have index zero.

The equivariant Nielsen number is trivial if and only if the indices of all $\mathrm{WH}$-fixedpoint classes for all the induced maps $f^{H}: X^{H} \rightarrow X^{H}$ are zero. This holds if and only if the indices for all the fixed-point classes of the maps $f^{H}$ are zero. This is equivalent to $R\left(f^{H}\right)$ is zero for all subgroups $H$ of $G$. Proposition 10.1 completes the proof.

It is unfortunate but necessary that the comparison in this section passes through the nonequivariant Reidemeister traces for isotropy subspaces. In particular, both the equivariant Nielsen number and the equivariant Reidemeister trace can be used to give lower bounds for the number of fixed points - see [31] for the Nielsen number and $[\mathbf{2 5}]$ for the Reidemeister trace - but this is lost with the classical Reidemeister trace. This essential incompatibility is unsurprising since these two approaches are fundamentally very different.

\section{References}

[1] J.F. Adams. Prerequisites (on equivariant stable homotopy) for Carlsson's lecture, in Algebraic Topology, Aarhus 1982 (Aarhus, 1982), volume 1051 of Lecture Notes in Math., pages 483-532. Springer, Berlin, 1984.

[2] R.F. Brown, The Lefschetz fixed point theorem. Scott, Foresman and Co., Glenview, Ill. London, 1971.

[3] M.C. Crabb, The homotopy coincidence index. J. Fixed Point Theory Appl., $7(1): 1-32,2010$.

[4] R.J. Daverman, Decompositions of Manifolds, volume 124 of Pure and Applied Mathematics, Academic Press, Inc., Orlando, FL, 1986. 
[5] T. Dieck, Transformation Groups, volume 8 of de Gruyter Studies in Mathematics, Walter de Gruyter \& Co., Berlin, 1987.

[6] A. Dold and D. Puppe, Duality, trace, and transfer, In Proceedings of the International Conference on Geometric Topology (Warsaw, 1978), pages 81102, Warsaw, 1980. PWN.

[7] E. Fadell and P. Wong, On deforming G-maps to be fixed point free, Pacific J. Math., 132(2):277-281, 1988.

[8] M. Groth, K. Ponto, and M. Shulman, The additivity of traces in monoidal derivators, J. K-Theory, 14(3):422-494, 2015, arXiv:1212:3377.

[9] J.R. Klein and B. Williams, Homotopical intersection theory, II, Equivariance, Math. Z., 264(4):849-880, 2010, arXiv:0803.0017v2.

[10] J.R. Klein and E.B. Williams, Homotopical intersection theory, I, Geom. Topol., 11:939-977, 2007.

[11] E. Laitinen and W. Lück, Equivariant Lefschetz classes, Osaka J. Math., 26(3):491-525, 1989.

[12] L.G. Lewis, Jr., J.P. May, M. Steinberger, and J.E. McClure, Equivariant stable homotopy theory, volume 1213 of Lecture Notes in Mathematics, SpringerVerlag, Berlin, 1986, With contributions by J.E. McClure.

[13] W. Lück and J. Rosenberg, The equivariant Lefschetz fixed point theorem for proper cocompact $G$-manifolds In High-dimensional manifold topology, pages 322-361, World Sci. Publ., River Edge, NJ, 2003.

[14] J.P. May, The additivity of traces in triangulated categories, Adv. Math., 163(1):34-73, 2001.

[15] J.P. May and J. Sigurdsson, Parametrized Homotopy Theory, volume 132 of Mathematical Surveys and Monographs, American Mathematical Society, Providence, RI, 2006.

[16] K. Ponto, Conicidence invariants and higher Reidemeister traces, To appear in J. Fixed Point Theory Appl. arXiv:1209. 3710.

[17] K. Ponto, Fixed point theory and trace for bicategories, Astérisque, (333):xii+102, 2010, arXiv:0807.1471.

[18] K. Ponto, Relative fixed point theory, Algebr. Geom. Topol., 11(2):839-886, 2011, arXiv:0906.0762.

[19] K. Ponto and M. Shulman, Shadows and traces in bicategories, J. Homotopy Relat. Struct., 8(2):151-200, 2013, arXiv:0910.1306.

[20] K. Ponto and M. Shulman, Duality and trace in symemtric monoidal categories, Expo. Math., 32(3):248-273, 2014, arXiv:1107.6032.

[21] K. Ponto and M. Shulman, Linearity of fixed point invariants, arXiv:1406.7861, 2014.

[22] K. Ponto and M. Shulman, Linearity of traces in monoidal categories and bicategories, arXiv:1406.7854, 2014.

[23] K. Ponto and M. Shulman, The multiplicativity of fixed point invariants, Algebr. Geom. Topol., 14(3):1275-1306, 2014, arXiv:1203.0950. 
[24] J. Stallings, Centerless groups - an algebraic formulation of Gottlieb's theorem, Topology, 4:129-134, 1965.

[25] G. Sunyeekhan, Equivariant Intersection Theory, ProQuest LLC, Ann Arbor, MI, 2010, Ph.D. thesis, University of Notre Dame.

[26] H. Ulrich, Fixed Point Theory of Parametrized Equivariant Maps, volume 1343 of Lecture Notes in Mathematics, Springer-Verlag, Berlin, 1988.

[27] J. Weber, The universal functorial equivariant Lefschetz invariant, $K$-Theory, 36(1-2):169-207 (2006), 2005.

[28] J. Weber, Equivariant Nielsen invariants for discrete groups, Pacific J. Math., 231(1):239-256, 2007.

[29] J.H.C. Whitehead, Simplicial spaces, nuclei and $m$-groups, Proc. London Math. Soc., s2-45(1):243-327, 1939.

[30] D. Wilczyński, Fixed point free equivariant homotopy classes, Fund. Math., 123(1):47-60, 1984.

[31] P. Wong, Equivariant Nielsen fixed point theory for G-maps, Pacific J. Math., 150(1):179-200, 1991.

[32] P. Wong, Equivariant Nielsen numbers, Pacific J. Math., 159(1):153-175, 1993.

Kate Ponto kate.ponto@uky.edu

Department of Mathematics, 719 Patterson Office Tower, Lexington, KY 40506-0027, USA 